\title{
1 HUWE1 employs a giant substrate-binding ring to feed and regulate its HECT E3 domain
}

3 Daniel B Grabarczyk ${ }^{1,4^{*}}$, Olga A Petrova ${ }^{1,4}$, Luiza Deszcz ${ }^{1}$, Robert Kurzbauer ${ }^{1}$, Paul Murphy ${ }^{1}$, Juraj

4 Ahel $^{1}$, Antonia Vogel ${ }^{1}$, Rebeca Gogova ${ }^{1}$, Victoria Faas $^{1}$, Darja Kordic ${ }^{1}$, Alexander Schleiffer ${ }^{1}$,

5 Anton Meinhart ${ }^{1}$, Richard Imre ${ }^{1}$, Anita Lehner ${ }^{2}$, Jana Neuhold ${ }^{2}$, Gerd Bader ${ }^{3}$, Peggy Stolt-

6 Bergner $^{3}$, Jark Böttcher ${ }^{3}$, Bernhard Wolkerstorfer ${ }^{3}$, Gerhard Fischer $^{3}$, Irina Grishkovskaya ${ }^{1}$,

7 David Haselbach ${ }^{1}$, Dirk Kessler ${ }^{3}$, and Tim Clausen ${ }^{1^{*}}$

$10{ }^{1}$ Research Institute of Molecular Pathology, Vienna BioCenter, Vienna, Austria

$11{ }^{2}$ Vienna Biocenter Core Facilities, Vienna BioCenter, Vienna, Austria

$12{ }^{3}$ Boehringer Ingelheim RCV GmbH \& Co. KG, Vienna, Austria.

$14{ }^{4}$ These authors contributed equally.

$15{ }^{*}$ Correspondence should be addressed to: Daniel Grabarczyk (daniel.grabarczyk@imp.ac.at) and

16 Tim Clausen (tim.clausen@imp.ac.at) 


\section{Abstract}

20 HUWE1 is a universal quality-control E3 ligase that marks diverse client proteins for

21 proteasomal degradation. Although the giant HECT enzyme is an essential component of the

22 ubiquitin-proteasome system closely linked with severe human diseases, its molecular

23 mechanism is little understood. Here, we present the crystal structure of Nematocida HUWE1,

24 revealing how a single E3 enzyme has specificity for a multitude of unrelated substrates. The

25 protein adopts a remarkable snake-like structure where the C-terminal HECT domain heads an

26 extended alpha solenoid body that coils in on itself and houses various protein-protein

27 interaction modules. Our integrative structural analysis shows that this ring structure is highly

28 dynamic, enabling the flexible HECT domain to reach protein targets presented by the various

29 acceptor sites. Together, our data demonstrate how HUWE1 is regulated by its unique

30 structure, adapting a promiscuous E3 ligase to selectively target unassembled orphan proteins. 


\section{Introduction}

33 E3 ligases regulate diverse cellular processes by conjugating ubiquitin, a small protein post-

34 translational modifier, to lysines of target proteins ${ }^{1}$. Not only do E3s have the critical role of

35 regulating which proteins are ubiquitinated and when, but they also team up with specific

36 partner E2s to determine whether a single ubiquitin or different types of polyubiquitin chain

37 are attached, where the linkage type determines the function of the mark ${ }^{2}$. Many E3 ligases

38 attach K48-linked chains to target proteins for degradation, either as a form of regulation or for

39 general protein quality control. Other linkage types and monoubiquitination have a range of

40 functions, for example in DNA repair, the immune system and protein trafficking. Thus to

41 understand a vast swathe of biology, we need to understand the specificity and mechanisms of

42 E3 ligases.

43 There are over 600 E3 ligases in humans, with some of the better understood ubiquitination

44 enzymes belonging to the RING family, including SCF ligases ${ }^{3}$ and the anaphase promoting

45 complex ${ }^{4}$. These are often large modular complexes with different subunits to specify the target

46 and regulate activity. In contrast, ligases of the HECT family usually act as a single polypeptide

47 chain, where the catalytic HECT domain is at the very C-terminus and the rest of the protein has

48 a regulatory and substrate recognition role ${ }^{5,6}$. The HECT E3 ligases are often very large, ranging

49 from 700 amino acids to giant enzymes of more than 3000 residues. Owing to their large size,

50 HECT enzymes are less well characterized than their RING counterparts with regards to

51 molecular mechanism, target selection and regulation ${ }^{7}$. Indeed, there is no structure of any full-

52 length HECT E3 ligase meaning that a critically important part of our biological understanding is

53 missing. 
54 One of the largest human HECT E3 ligases is HUWE1 (4374 residues, 482 kDa), an essential

55 protein which regulates a myriad of biological processes through ubiquitination ${ }^{8}$ (Fig. 1a). The

56 giant E3 initially gained the spotlight as a specific regulator of many key cellular factors,

57 although the exact ubiquitin chain-types and downstream effects are not always clear. The

58 targeted substrates include apoptosis factors like $\mathrm{Mcl}-1^{9}, \mathrm{Miz}^{10}$ and $\mathrm{p} 53^{11}$, proliferation and

59 developmental factors like $\mathrm{c}-\mathrm{Myc}^{12}, \mathrm{AsCl}^{-13} \mathrm{1}^{13}, \mathrm{FOXP3}^{14}$ and $\mathrm{Dvl} \mathrm{I}^{15}$, and DNA replication and repair

60 proteins like $C d c 6^{16}$, Pol $\beta^{17}$ and BRCA $1^{18}$. Moreover, it is increasingly clear that HUWE1 also has

61 a general protein quality control function, whereby it corrects proteome imbalances by acting

62 in the unassembled soluble protein degradation pathway (USPD, ${ }^{19}$ ). Here, the E3 enzyme

63 targets 'orphan' proteins, those missing their complex partners, for proteasomal degradation,

64 particularly the unassembled subunits of macromolecular assemblies, like ribosomes ${ }^{20}$ or

65 nucleosomes ${ }^{21,22}$. Such proteome imbalances are likely to occur in cancer cells ${ }^{23}$, most notably

66 in aneuploid cells ${ }^{24}$. Considering that many HUWE1 targets are involved in cancer-related

67 processes and that HUWE1 itself is frequently mutated in cancers ${ }^{25}$, the giant HECT enzyme is a

68 highly attractive target for therapeutic intervention ${ }^{26}$. Additionally, mutation of HUWE1 is

69 associated with severe X-linked intellectual disability ${ }^{27}$, further highlighting the urgent need to

70 unravel the function of this enigmatic E3 ligase at a molecular level.

71 It is puzzling how one polypeptide can regulate so many distinct targets, ranging from specific

72 proliferation factors to theoretically any misassembled complex subunit. The huge E3 portion

73 preceding the HECT domain is likely to hold the answer to this, but is currently largely

74 uncharacterized except for a few known protein-interaction modules, such as a poly-ADP-ribose

75 interaction WWE domain (PDB 6MIW), various ubiquitin binding domains (PDB 2MUL, 2EKK) 
76 and an $\mathrm{Mcl}-1$ interacting $\mathrm{BH} 3$ motif (PDB $5 \mathrm{C} 6 \mathrm{H}$ ). In order to understand the promiscuous

77 specificity and the regulation of HUWE1, we used the compact protein from Nematocida as a

78 model system (HUWE1 $1_{N}, 2490$ residues, $\left.287 \mathrm{kDa}\right)$, reconstituted the full-length protein in

79 functional form and determined its crystal structure. Accordingly, HUWE1 forms a snake-like

80 structure with a large alpha-solenoid body headed by the HECT domain. An integrative

81 structural biology analysis shows that the head and body of the giant protein are highly

82 dynamic structures, explaining how the catalytic HECT domain can ubiquitinate substrates

83 docked around the coiled ring. Our data suggest that an evolutionarily conserved function of

84 HUWE1 is as a quality control enzyme for misassembled complexes, and we show which

85 structural features of HUWE1 are required for targeting substrates and regulating the HECT

86 domain.

87 
Results

Nematocida $\mathrm{HUWE}_{\mathrm{N}}$ as model system

90 Due to its large size, human HUWE1 is a challenging protein for biochemical and structural

91 analysis. Thus, most in vitro studies so far are limited to its isolated C-terminal HECT domain, a

92 small fraction of the full protein (40 kDa from $482 \mathrm{kDa}$ ). To obtain well-behaving full-length

93 HUWE1 we searched for a more compact variant. To this regard, the smaller $374 \mathrm{kDa}$ budding

94 yeast orthologue, Tom1, has been shown to have similar functions as HUWE1 in marking DNA

95 replication factors, histone proteins and unassembled ribosomal proteins for proteasomal

96 degradation ${ }^{16,20,22}$. We found an even shorter fungal orthologue, HUWE1 $1_{N}(287 \mathrm{kDa})$, from the

97 parasite Nematocida sp. ERTm5. Full-length HUWE1 $1_{N}$ could be produced in large amounts and

98 in a functional form in insect cells, enabling a detailed structure-function analysis.

99 To demonstrate that Huwe $1_{N}$ is a bona fide orthologue of human HUWE1, we tested its activity

100 on a panel of known in vivo substrates (Fig. 1b). In the presence of UbcH5b, HUWE1 ${ }_{\mathrm{N}}$ strongly

101 ubiquitinated the transcription factor FOXP3, histone $\mathrm{H} 1$ and histone $\mathrm{H} 2 \mathrm{~B}$, generating ladders of

102 ubiquitinated species in each case. No activity was seen against Mcl-1, but it should be noted

103 that the Mcl-1 binding site identified in human HUWE1 is not conserved in the fungal HUWE1 ${ }_{N}$.

$104 \mathrm{~A} \mathrm{HUWE}_{\mathrm{N}}-\mathrm{CA}$ variant with the catalytic cysteine mutated was inactive, confirming the activity

105 we observed was due to specific E3 activity (Fig. 1b). Autoubiquitination by HUWE1 $1_{N}$ as well as

106 free di-ubiquitin generation were rather weak, but these activities were stimulated by addition

107 of substrate, hinting that the catalytic activity of $H U W E 1_{N}$ is regulated by substrate binding (Fig.

108 1b). To determine the types of ubiquitin chains generated by HUWE $1_{N}$ we performed a mass 
spectrometry analysis of the ubiquitinated products (Fig. 1c). These data reveal the

110 predominance of $\mathrm{K} 48, \mathrm{~K} 6$ and $\mathrm{K} 33$-linked chains, consistent with previous reports for human

111 HUWE1 $1^{28,29}$, and suggesting that ubiquitination by HUWE1 $1_{N}$ has a degradative function.

112 The best substrates that we identified in a screen of reported in vivo targets were positively

113 charged proteins with an isoelectric point $(\mathrm{pl})>9$, and missing their complex partners. To test

114 whether $\mathrm{HUWE} 1_{\mathrm{N}}$ recognizes the orphan nature of these proteins, we first compared

115 ubiquitination of the isolated ribosomal protein $\mathrm{Rp} / 26^{20}$ with intact ribosomes. At the same

116 molar concentration of substrate, we observed clear ubiquitination of the orphan subunit, but

117 no ubiquitination of proteins integrated into the ribosome (Fig. 1d). Similarly, histone H2B was

118 readily ubiquitinated but intact nucleosomes were not (Fig. 1e). We next directly tested

119 whether HUWE1 $1_{N}$ recognizes surfaces normally blocked by protein or nucleic acid interactions

120 using the simple system of histone $\mathrm{H1}$, which is integrated into the nucleosome only through

121 protein-DNA interactions (Fig. 1f). Four-way junction (4WJ) DNA mimics this interaction with

122 sub-nanomolar affinity ${ }^{30}$. We titrated this into the reaction and observed complete loss of

123 histone $\mathrm{H} 1$ ubiquitination by $\mathrm{HUWE}_{\mathrm{N}}$ at a $1: 1$ ratio of histone $\mathrm{H} 1$ and 4 WJ DNA (Fig. 1f). Thus,

$124 \mathrm{HUWE}_{\mathrm{N}}$ recognises portions of histone $\mathrm{H} 1$ which are normally shielded by DNA. To provide

125 further evidence of this targeting mechanism we mapped the ubiquitination sites of Rpl26 by

126 mass spectrometry (Fig. 1g). These data reveal that many of the modified lysines would be

127 occluded within the context of the intact ribosome and are thus only accessible for the

128 dissociated Rpl26. Finally, we tested whether these substrate recognition principles are

129 conserved in metazoan HUWE1. In these experiments, we used the $C$. elegans orthologue

130 (CeHUWE1), which could be functionally produced using insect cell expression, as a model 
131 system. CeHUWE1 also strongly ubiquitinated Rpl26 and histone H1, and the ubiquitination of

132 histone $\mathrm{H} 1$ was also blocked by 4WJ DNA (Fig. 1h). In conclusion, our data show that the

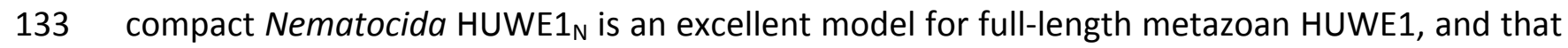

134 one evolutionary conserved function of this giant enzyme is to mark unassembled orphan 135 proteins for degradation.

\section{Determining the crystal structure of HUWE1 $\mathbf{N}_{\mathrm{N}}$}

137 To reveal how the structurally uncharacterized 2100 amino acid $\mathrm{N}$-terminal region of HUWE1 138 contribute to E3 function and regulation, we attempted to crystallize full-length Huwe $1_{\mathrm{N}^{-}} C A$.

139 After cleaving the N-terminal purification tag, we obtained crystals that diffracted 140 anisotropically to $3.05 \AA$ resolution (Table S1). We were able to partially solve the phase

141 problem by molecular replacement using the human HUWE1 HECT domain as search model. To

142 build the backbone of the large $\mathrm{N}$-terminal region, we used an experimental density map that

143 we obtained by collecting anomalous diffraction data from crystals soaked with $\mathrm{Ta}_{6} \mathrm{Br}_{14}$ clusters

144 or grown with selenomethionine-substituted protein. Guided by the numerous Se sites

145 identified in the anomalous map (Fig. S1a), and contacts predicted by the RaptorX server ${ }^{31}$, we

146 were able to build an atomic model of the two $H U W E 1_{N}$ proteins present in the asymmetric

147 unit of the HUWE1 $1_{N}$ crystals (Fig. S1b). We could model almost the entire polypeptide chain, 148 except two prolonged intrinsically disordered regions (IDR: residues 1442-1521 and 1576-1670), 149 the UBM domain (1671-1764) and several flexible loops connecting helical repeats (4-13, 199$150207,1210-1239,1828-1850$ and 2483-2490). Ultimately, the crystal structure was refined to a 
151 final $R_{\text {free }}$ of $23.3 \%$ and Molprobity score of $2.35^{32}$. The final electron density was of excellent

152 quality, revealing side-chain conformations for most residues (Fig. S1c, Video S1).

\section{HUWE1 is a ring forming HECT ubiquitin ligase}

154 In our crystal structure, HUWE1 $1_{N}$ adopts a snake-like scaffold composed of an N-terminal tail 155 (residues 1-349), an elongated body (350-1441), a neck region (1765-2114) and the head - the 156 HECT domain (2115-2490) (Fig. 2a). The tail, body and neck, preceding the HECT domain, 157 together form an $\alpha$-solenoid structure, composed of 34 Armadillo-like (ARM) repeats (Fig. S2a).

158 This coils in on itself to form a giant ellipsoidal ring, measuring approximately $140 \AA$ by $90 \AA$, 159 and an inner diameter of about $60 \AA$ (Fig. 2bc). Rather than being a simple repeat structure, the $160 \alpha$-solenoid is distorted and decorated by four insertions (Ins1-4) (Fig. 2a-c). The HECT is 161 connected to the rest of the structure through the deformed final helix of repeat ARM34, which 162 packs loosely on helix $\alpha 1$ of the HECT domain. ARM34 was previously assigned as the 'thumb' 163 and 'pointer' helices in the crystal structure of an N-terminally extend HECT domain ${ }^{33}$.

164 Considering the large difference in sequence length, we also tested if metazoan HUWE1 has a 165 similar architecture. For this, we performed negative stain EM analysis on the 4177 amino acid 166 CeHUWE1. The resulting low-resolution 3D model showed high similarity to Huwe $1_{N}$ in both its

167 dimensions and overall shape (Fig. 2d). Likewise, the recently reported cryo-EM structure of 168 human HUWE1 shows a ring-shaped structure with similar diameter and domain architecture ${ }^{34}$. 169 We thus propose that the core structure of HUWE1 is conserved and that the additional 17001702000 residues of metazoan orthologues constitute flexible appendices. The insertions include 171 several previously characterised domains of human HUWE1 which are not present in Huwe $1_{\mathrm{N}}$ : 
172 the ubiquitin association domain (UBA) and the ubiquitin interaction motif (UIM), a

173 polyADPribose-interacting WWE domain and an $\mathrm{Mcl}-1$ interacting $\mathrm{BH} 3$ motif. Considering that

174 these features would be positioned around the solenoid body of HUWE1 (Fig. 2e, Fig. S2b), an

175 appealing mechanism emerges for how HUWE1 may function: the ring could act as a substrate

176 binding platform decorated by different docking sites, which could feed proteins to the HECT

177 domain. Additionally, ARM repeat proteins frequently bind peptides within their interior ${ }^{35,36}$

178 (Fig. S3a), suggesting that the solenoid itself could act as a generic interaction surface. In 179 support of this model for HUWE1 function, reported intellectual disability mutations are not 180 just located on the HECT domain, but also on the solenoid and the inserted domains (Fig. S2c).

\section{Functional insertions within the $\mathrm{HUWE} \mathbf{1}_{\mathrm{N}}$ ring}

182 The four insertions within the solenoid form unusual structures which link together different 183 parts of $\operatorname{HUWE}_{\mathrm{N}}$ (Fig. 2b, 3a-c). Insertion 1 (Ins1) causes a sharp bend of the solenoid 184 structure. It consists of a helix and flexible $\beta$-hairpin-like loops between ARM4 and ARM5 185 (Fig. 3a) and forms part of the interface between the tail and neck. Ins2 is a three-helix bundle 186 between ARM23 and ARM24 resting on top of the solenoid on the opposite side of the ring 187 relative to the HECT domain (Fig. 3b). Curiously, Ins3 (residues 1442-1764), positioned between 188 ARM27 and ARM28, backtracks three ARM repeats, such that three linearly arranged helices 189 (Ins3-3H) can interact closely with Ins2 and the ARM repeats beneath, yielding a composite 190 portion of the solenoid backbone (Fig. 3b). Ins3-3H is sandwiched between two conserved 191 highly acidic unstructured regions. Ins3-IDR1 runs from 1442-1521, contains 41\% Glu/Asp 192 residues and has a predicted pl of 2.7, while Ins3-IDR2 is between $1576-1670$ and contains $45 \%$ 
193 Glu/Asp contributing to a pl of 3.6. At the C-terminal end of Ins3 lies the unresolved ubiquitin

194 binding UBM domain (Ins3-UBM, 1671-1764). Importantly, the "empty" ring-like structure as

195 observed in the electron density map may be misleading. The unusual topology of Ins3 (Fig. 3b),

196 crossing the plane of the solenoid in a defined manner, implies that the ring is filled with the

197 disordered IDR1 and IDR2 segments generating a 173-residue mesh of negatively charged

198 residues in the center of the HUWE1 solenoid. The final insertion, Ins4 (residues 2055-2076),

199 forms a $\beta$-hairpin between ARM33 and ARM34, protruding towards the HECT domain (Fig. 3c).

200 To determine whether these inserted features contribute to the specificity or regulation of the

201 HECT domain, we generated four deletion variants to remove the inserts without affecting the

202 Armadillo backbone - HUWE1 ${ }_{N}-\Delta I n s 2$ (1146-1243 replaced by GSSG), HUWE1 ${ }_{N}-\Delta$ Ins4 (2057-

2032070 replaced by GSSG), HUWE $1_{N}-\Delta 1500-1660$ (removing ins3-3H, and parts of the two IDRs)

204 and $H U W E 1_{N}-\triangle U B M$ (1671-1750 replaced by GGSGG). We first tested the variants for specific

205 substrate ubiquitination using histone $H 1$, as well as for presumably unspecific

206 autoubiquitination (Fig. 3d). The Ins4 and UBM deletions had only minor effects on

207 ubiquitination in these assays. In contrast, both the HUWE $1_{N^{-}} \Delta 1500-1660$ and the $H U W E 1_{N^{-}}$

208 Alns2 variants showed much lower histone $\mathrm{H} 1$ ubiquitination activity than the wild-type

209 enzyme. This was not due to a decrease in the catalytic activity of these variants, because their

210 substrate-independent autoubiquitination activity was not reduced. In fact, this activity was

211 much stronger for HUWE $1_{N}-\triangle 1500-1660$ than for the wild-type E3 (Fig. 3e). Together, these

212 data suggest that the topologically unusual interaction between Ins 2 and Ins 3 is crucial for both

213 the specific recognition of substrates and for inhibiting the basal autoubiquitination activity of

214 the enzyme. This could be either because the Ins2-Ins3 interaction maintains the integrity of 
215 the ring structure or because it correctly positions the acidic IDR1/IDR2 regions. We next

216 replaced either IDR1 (1442-1522) or IDR2 (1610-1670) with an 18-residue glycine-serine linker

217 to probe the importance of these regions without disrupting the ins2-ins 3 interaction. While

218 IDR2 deletion showed no effect, the $\triangle I D R 1$ variant displayed greatly reduced histone $\mathrm{H} 1$

219 ubiquitination and increased autoubiquitination (Fig. 3de). Thus, IDR1 plays an important role

220 in orphan substrate recognition and HECT regulation.

\section{Dynamic interfaces stabilize the $\mathrm{HUWE}_{\mathrm{N}}$ ring}

222 Unlike most $\alpha$-solenoid proteins, Huwe $1_{N}$ coils tightly enough to form a closed ring with three

223 non-contiguous contacts between the N-terminal tail and C-terminal neck portions (Fig. 2b, 4a):

224 The Ins1-ARM27, ARM5-ARM29 and ARM6-ARM33 interfaces are small (total surface area of

$225654 \AA^{2}$ ), contain few specific interactions, and involve loop regions with elevated thermal

226 motion factors (B-factors), suggesting that the interfaces are rather dynamic and flexible. The

227 small Ins1-ARM27 interface mostly consists of van der Waals contacts that occur between

228 aliphatic side chains of various residues, organized around Y177 (Fig. 4b). However, this site

229 may also comprise additional regions not well resolved in our structure, such as residues 1828-

230 1850. The ARM5-ARM29 interface is larger and established by electrostatic interactions with

231 potential salt bridges between R257 and E1855 plus D251 and R1792, as well as putative

232 hydrogen bonding interactions between S252 and E1863 plus S256 and E1900 (Fig. 4c). The

233 most clearly resolved interaction is between ARM6 and ARM33 and involves H303 hydrogen

234 bonding with S2040, stacking interactions with Y2038 and a probable salt bridge between E304

235 and $\mathrm{H} 1984$ (Fig. 4d). 
236 To dissect the function of these interfaces, which belong to the most conserved peptide

237 stretches in the HUWE1 protein family (Fig. S3b), we designed site-specific mutations to disrupt

238 them. However, both the variants we attempted to purify, $H 303 A / E 304 A$ and $D 251 R$, were

239 unstable $\mathrm{HUWE} 1_{\mathrm{N}}$ proteins prone to precipitation. This suggests that closure of the ring through

240 these interfaces is necessary for stabilizing the HUWE1 $1_{N}$ structure.

241 HUWE1 combines a flexible substrate-binding ring with a mobile HECT

242 For the HECT domain to ubiquitinate substrates bound to different parts of the ring, a high

243 degree of flexibility would be required. Indeed, comparison of the two copies of HUWE1 $1_{N}$ in the

244 asymmetric unit shows a certain breathability of the ARM repeats (Fig. S3c). Therefore, we

245 sought to determine how this structure behaves in solution. Cross-linking mass spectrometry of

$246 \mathrm{HUWE}_{\mathrm{N}}$ resulted in several cross-links between the $\mathrm{N}$-terminus and $\mathrm{C}$-terminus of the protein

247 (Fig. 5a, Fig. S4), showing that the ring-like structure is the prevalent conformation in solution.

248 Additionally, cross-links were detected between Ins2 and Ins3-3H, confirming the composite

249 topology of this region. Notably, the C-lobe of the HECT domain, housing the catalytic cysteine,

250 formed cross-links with sites throughout the solenoid ring (Fig. 5a, Fig. S4). This suggests that

251 the HECT domain has sufficient flexibility to reach substrates bound to any part of the solenoid.

252 To probe the conformational flexibility of $\mathrm{HUWE}_{\mathrm{N}}$ at higher resolution we turned to cryo-EM.

253 Classification of the particles revealed that the E3 ligase is highly dynamic in its ring structure

254 and adopts a continuum of coiled conformations ranging from closed to fully open (Fig. S5). We

255 refined two classes at $5.3 \AA$ (Class 1 ) and $6.4 \AA$ (Class 2 ) resolution (Fig. 5b, Table S2), allowing

256 us to unambiguously dock the secondary structure of our model (Fig. S6). Compared to the 
257 crystal structure, the cryo-EM structures exhibited a striking relaxation over the entire length of

258 the solenoid, leading to breaks and shifts in the interfaces between the neck and tail (Fig. $5 \mathrm{~cd}$ ).

259 In the slightly opened Class 1, Ins1 still stabilizes the ring-like structure but now its interaction

260 has been shifted from ARM27 to ARM28. Further along, ARM5 now interacts with ARM33

261 instead of ARM29, while ARM6 has lost all interactions. In contrast, in Class 2, the solenoid is

262 opened and shows no visible contacts between tail and neck. Together, our structural data

263 demonstrate a high level of plasticity and dynamicity in the intramolecular contacts which

264 maintain the overall architecture of the ring but allow for structural flexibility. The adaptable

265 ring structure is enabled by the small size of the interfaces, the lack of hydrophobic interactions

266 and the flexibility of involved motifs (Fig. 4e). This dynamicity could be critical to allow large-

267 scale movements of the HECT domain and to properly position the various substrate binding

268 sites. In fact, the inherent en-bloc flexibility of the HECT domain was clearly seen in the EM

269 densities, as the domain was less well-resolved than the rest of the structure (Fig. S5, Video S2,

270 S3).

271 The HECT domain is inhibited in full-length HUWE1

272 The HECT domain was present in a similar conformation as previously published human HUWE1

273 structures ${ }^{33,37}$. The E3 active site is located on the face of the HECT domain that is opposite to

274 the substrate-capturing solenoid ring (Fig. 6a). Accordingly, in the first step of the ligation

275 reaction, the E2-Ub complex can bind and transfer Ub to the HECT catalytic cysteine without

276 interference from the solenoid ring, as seen when superimposing the structure of a HECT/E2-

277 Ub complex (Fig. 6b) ${ }^{38}$. Accessing substrates bound to the ring would require a $180^{\circ}$ rotation of 
278 the HECT C-lobe. In fact, such a movement is expected in the second step of the reaction, as

279 seen for a trapped HECT-Ub/substrate complex ${ }^{5}$. However, when this state is overlaid on the 280 HUWE $1_{N}$ crystal structure, there is a steric clash between the predicted position of the C-lobe

281 and the solenoid body of HUWE1 $1_{N}\left(\right.$ Fig. 6c). We thus presume that the crystallized HUWE1 $1_{N}$

282 represents the latent E3 state, in which the solenoid ring presents a steric block preventing

283 substrate ubiquitination. When we instead overlay the HECT-Ub/substrate complex on our

284 open Cryo-EM Class 2, these clashes are relieved (Fig. S7a), suggesting that a conformational

285 rearrangement of the ring could be linked to HECT domain regulation. Another interesting

286 comparison is with the previous structure of the $\mathrm{N}$-terminally extended HECT domain of human

287 HUWE1 ${ }^{33}$. The 'pointer' and 'thumb' helices equivalent to ARM34 in our structure have

288 remarkably different structural organisation, which also differs in the two copies present in the

289 reported crystal form (Fig. S7b). Given its structural plasticity and immediate proximity to the

290 HECT domain, ARM34 could serve as a molecular hinge controlling the orientation of the

291 catalytic domain. Moreover, it was shown that mutation of F3892A, a central hinge residue

292 equivalent to Huwe $1_{N} F 2105$, causes a strong increase in activity in vivo ${ }^{33}$. A closer look at our

293 full-length structure reveals such a mutation could greatly increase the flexibility of the hinge

294 region, thus bypassing steric inhibition imposed by the ring (Fig. S7c).

295 To understand how the HECT is regulated in the context of full-length HUWE1 $1_{N}$, we tested the 296 activity of the isolated HECT domain (residues 2100-2490). For this construct, we observed very

297 low ubiquitination activity on the Rpl26 substrate (Fig. 6d), which, in contrast, is strongly

298 ubiquitinated by the full-length protein. This is consistent with the idea that the solenoid ring,

299 and its insertions, are critical for substrate targeting. Importantly, these data also demonstrate 
300 the necessity of using full-length enzymes to understand the substrate specificity of HECT E3

301 ligases. Moreover, the isolated HECT domain autoubiquitinated itself much more efficiently 302 than the full-length enzyme, resulting in extended poly-ubiquitin chains (Fig. 6e). This suggests 303 that, in the full-length protein, the solenoid inhibits the non-specific activity of the HECT 304 domain, both in terms of substrate recruitment and substrate poly-ubiquitination. We observed 305 similar behaviour for the metazoan CeHUWE1, where the isolated HECT domain rapidly 306 depleted ubiquitin to generate high molecular weight ubiquitinated species (Fig. S7d), pointing 307 to a common auto-regulation mechanism.

308 To dissect how the non-specific activity of the HECT is inhibited, and how the full-length protein

309 is activated by substrates (Fig. 1b), we used single turnover assays following discharge of 310 ubiquitin-loaded E2 onto excess HUWE1 (Fig. 6f). Consistent with a previous report for the 311 human HUWE1 HECT domain ${ }^{37}$, the discharge activity was slow and not complete within an 312 hour. The reaction resulted in accumulation of thioester-loaded HUWE1 $1_{N}$ (Fig. 6f), as indicated 313 by the sensitivity of the attached ubiquitin to chemical reduction (Fig. 6f). In the presence of 314 the histone $\mathrm{H} 1$ substrate, the rate of discharge increased markedly, resulting in complete loss of 315 the E2-Ub signal within an hour (Fig. 6f). This suggests substrate-induced activation occurs at 316 the thioester transfer step. In contrast, when we used the isolated HECT domain, the rate of 317 discharge remained very slow (Fig. 6f). However, here the ubiquitinated HECT species were 318 insensitive to reduction pointing to isopeptide-linked, rather than thioester-linked ubiquitin 319 species (Fig. 6f). Together, these findings indicate that in the absence of the solenoid the 320 thioester-loaded HECT is rapidly discharged by autoubiquitination. Thus, our data highlight the 321 complex regulation of this promiscuous E3 ligase at various steps of the ubiquitination reaction. 


\section{Discussion}

323 The giant E3 ligase HUWE1 adopts an intricately coiled structure comprising a ring-shaped,

324 solenoid body serving as substrate binding platform and an attached HECT domain that

325 ubiquitinates bound proteins within its radius of manoeuvre. Aside from the characterized

326 docking sites for poly-ADP-ribose, ubiquitin and Mcl-1 (Fig. 2e), the ring-shaped solenoid likely

327 harbours further recognition modules, for example ligand binding sites within its ARM repeats.

328 Intriguingly, our data show that the IDR1 region, a segment of the Ins3 appendix, constitutes an

329 Asp/Glu-rich molecular mesh in the centre of the solenoid, used to recognize histone $\mathrm{H} 1$ and

330 presumably other positively charged proteins (Fig. 3). The architecture of HUWE1, combining a

331 multivalent docking platform with a flexible catalytic head, presents an alternative E3 strategy

332 compared to the prominent class of SCF RING ligases ${ }^{3}$. These enzymes use a modular setup

333 where promiscuity is achieved through exchangeable F-box protein adaptor modules to target

334 different substrates. The advantage of using a large platform with multiple substrate docking

335 sites, like HUWE1, is that it can act as a general-purpose E3 enzyme ready for all cell states. If

336 additional regulation is required, one attractive possibility is that the individual docking sites

337 may be post-translationally modified to favour or disfavour ubiquitination of certain substrates.

338 Such regulated substrate targeting has been reported for Atoh1 (a transcription factor involved

339 in cerebellar development) and the Wnt pathway component Dvl, both of which are

340 ubiquitinated by HUWE1 in a phospho-dependent manner ${ }^{15,39}$.

341 How could the HECT domain reach all these substrates? Our integrative structural biology data

342 show that the overall HUWE1 architecture is highly dynamic and flexible. The ring can adopt an

343 entire range of conformations, opened to different extents, with this plasticity supported by the 
344 use of a multitude of small interfaces with low specificity. These large-scale movements are

345 then likely to be combined with flexibility in orientation of the HECT domain relative to the 346 neck. As compared to SCF complexes, where the catalytic RING domain is connected by a

347 flexible peptide linker to a rigid scaffold ${ }^{40}$, the HUWE1 architecture offers an alternative 348 solution to ubiquitinate a variety of substrate proteins in a reaction sphere. In HUWE1, the 349 HECT domain is enabled to scan a series of docking sites arranged in a defined radius along the 350 ring-shaped scaffold.

351 Our data suggest that the huge $\mathrm{N}$-terminal ring of HUWE1 $1_{N}$ is not only responsible for substrate 352 specificity but also regulates the activity of the HECT domain. One possible way this could be 353 achieved is that the large conformational changes required during the catalytic cycle are 354 blocked by the N-terminal region of the solenoid (Fig. 6c). Alternatively, there could be a direct 355 allosteric effect on the catalytic HECT domain, promoted by the intricate connection between 356 ARM34 and the HECT $\alpha 1$ helix. Interestingly, a previous study showed that removing the $\alpha 1$ 357 helix increases the rate of thioester transfer to HUWE1 ${ }^{37}$, which is similar to the effect we 358 observe for our substrate-induced activation (Fig. 6f). Auto-inhibition of a HECT by its N359 terminal region has also been reported for WWP $2^{6}$, and may represent a general strategy for 360 this class of ubiquitination enzymes. In addition to linking substrate binding with E3 activation, 361 the observed ring structure offers a way to regulate the E3 activity of HUWE1 in trans. By 362 modulating the transition of open and closed states, respectively, regulatory proteins could act 363 as inhibitory or activating factors that adjust the activity of HUWE1 to the needs of the cell.

364 In conclusion, the unique architecture of HUWE1 explains its remarkable ability to function as a 365 promiscuous E3 ligase, targeting a broad range of unassembled orphan proteins as well as 
366 diverse regulatory proteins. Of note, further giant ubiquitin ligases like UBR4, HECTD1 and 367 Mycbp2 exhibit similar non-catalytic scaffolds, being composed of extended ARM repeats 368 interrupted by diverse protein-protein interaction motifs. Thus, HUWE1 can be a paradigm for 369 understanding how full-length E3 ligases target their substrates and adapt their activities within 370 the complex ubiquitin-proteasome system. With the Nematocida model system, we now can 371 explore the molecular details of how HUWE1 is regulated. These studies will provide the 372 structural and mechanistic framework to rationally develop HUWE1 directed therapeutics, 373 including small-molecule degraders that could exploit the promiscuity of an essential E3 ligase 374 to induce proteasomal degradation of diverse neo-substrates. 
377 We thank all members of the Clausen group for remarks on the manuscript and discussions, 378 and the Mass Spectrometry and ProTech services from the Vienna BioCenter Core Facilities for 379 their support. We are grateful to the scientific staff at the beamline X10SA, Paul Scherrer 380 Institute (Villigen, Switzerland), as well as the beamline P11, DESY (Hamburg, Germany) for 381 support in crystallographic data collection. We acknowledge the following cryo-EM facilities for 382 their access and support: CEITEC MU of CIISB, Instruct-CZ Centre (proposal LM2018127), UK's 383 national Electron Bio-imaging Centre (Diamond Lightsource, proposal EM BI25222), EM facility 384 at the Institute of Science and Technology (IST) Austria, and the EM facility at the Vienna 385 BioCenter Core Facilities (VBCF). We also acknowledge members of the Protein Science lab at 386 Boehringer Ingelheim for support with expression and purification of HUWE $1_{N}$, and Victor387 Valentin Hodirnau for help with the cryo-EM analysis. The project has received funding from 388 the European Research Council (ERC) under the European Union's Horizon 2020 research and 389 innovation programme (AdG 694978) and Marie Curie Skłodowska Grant Agreement Nr. 390 847548, an FFG Headquarter Grant (No 852936) and the Austrian Science Fund (FWF, SFB F 79).

391 P.M. and O.A.P are members of the Boehringer Ingelheim Discovery Research global post-doc 392 program. The IMP is supported by Boehringer Ingelheim.

\section{Conflict of Interest}

395 There is no conflict of interest. 
397 Coordinates of the HUWE1 $1_{N}$ crystal structure have been deposited at the Protein Data Bank 398 (PDBe) under accession code 7BII. Cryo-EM maps and atomic coordinates have been deposited 399 in the EMDB with accession codes EMD-12318 and EMD-12319 and PDB under 7NH1 and 7NH3.

\section{Author contributions}

401 D.B.G. and T.C. designed experiments; L.D., O.A.P., D.B.G., R.K., P.M., R.G., V.F., D.K., A.L., J.N. 402 prepared expression constructs, purified HUWE1 and performed biochemical assays; O.A.P., 403 I.G., J.A., D.H. performed the cryo-EM analysis, A.V., R.I. the XL-MS analysis, A.S. the 404 bioinformatic analysis, D.B.G., T.C., A.M., G.B., P.S.B., J.B., B.W., G.F., D.K. the crystallographic 405 analysis; D.B.G. built the molecular models; D.B.G. and T.C. coordinated the research project 406 and prepared the manuscript with inputs from all authors. 
Figures

409

410 Figure $1-$ HUWE1 $_{N}$ targets orphan proteins for degradation. (a) Cartoon depicting the domain

411 organization and functional roles of HUWE1. (b) HUWE1 ${ }_{N}$ substrate screen. E1-E2-E3

412 ubiquitination assays were performed using $4 \mu \mathrm{M}$ of the indicated substrates and either wild-

413 type $H U W E 1_{N}$ or the inactive $H U W E 1_{N}-C A$ variant. The ubiquitinated products were visualized

414 by using ubiquitin labeled with the DyLight488 fluorophore. (c) Linkage specificity of HUWE1 $1_{N}$ as

415 seen in autoubiquitination. Ubiquitin chains with the indicated linkage types were detected by

416 mass spectrometry. The ratio of ubiquitinated to total detected peptides for each linkage type

417 is shown as a percentage (d-e) Ubiquitination of an orphan ribosomal subunit and an orphan

418 nucleosome subunit, respectively. Assays were performed with $500 \mathrm{nM}$ substrate and only

419 show the region of the gel containing ubiquitinated substrates, which were visualized with Ub-

420 DyLight800 in (c) and Ub-DyLight488 in (d). The position of Rpl26 (colored red) within the

421 ribosome (PDB 6T59) and histone H2B (colored red) within the nucleosome (PDB 3AFA) are

422 displayed with nucleic acids colored yellow. (f) Histone H1 ubiquitination is blocked by 4WJ

423 DNA. Assays were performed for 120 minutes. (g) An Rpl26 ubiquitination reaction was

424 analyzed by mass spectrometry. Detected ubiquitinated lysines are indicated in the context of

425 the ribosome (PDB 6T59). (h) Ubiquitination of orphan proteins by CeHUWE1, using $2 \mu \mathrm{M}$

426 substrate and $4 \mu \mathrm{M} 4 \mathrm{WJ}$ DNA in the last lane, and visualized with Ub-DyLight800. All uncropped

427 and Coomassie-stained gels are shown in Fig. S8. 
429 Figure 2 - Overall architecture of the giant E3 ligase HUWE1 $1_{N}$. (a) Domain architecture of

$430 \mathrm{HUWE} 1_{\mathrm{N}}$. ARM repeats 1-34 are numbered, with the four insertions indicated. The position of 431 human HUWE1 insertions, absent in HUWE1 $1_{N}$, are shown in square brackets. (b-c) Crystal 432 structure of $H U W E 1_{N}$. The structure is shown in cartoon representation from four different 433 views, using the same color code as in a (catalytic Cys in red). A schematic cartoon illustrates 434 the snake-like organization of the E3 ligase. (d) Negative stain EM analysis of CeHUWE1. The 435 obtained EM density is shown from two viewpoints with approximate dimensions indicated. 436 HECT and tail domains are labelled based on homology to HUWE1 $1_{N}$. (e) Organization and 437 increasing complexity of HUWE1. 
439 Figure 3 - Functional insertions within the HUWE $1_{N}$ ring. (abc) Molecular details of insertions

440 Ins1, Ins2, Ins3 and Ins4 within the alpha-solenoid. (d) Histone H1 ubiquitination assay of the

441 HUWE1 $_{\mathrm{N}}$ insertion deletion variants. Ubiquitination of $4 \mu \mathrm{M}$ histone $\mathrm{H} 1$ was visualized by

442 fluorescence with Ub-DyLight488. (e) Autoubiquitination activity of the HUWE1 $1_{N}$ variants in the 443 absence of a substrate. Only the region of the gel with HUWE1 $1_{N}-U b^{*}$ band is shown.

444 
445 Figure 4 - Dynamic interfaces stabilize the $\mathrm{HUWE}_{\mathrm{N}}$ ring. (a) Overview of the interface 446 between the tail and neck regions of $\mathrm{HUWE}_{\mathrm{N}}$. A cartoon schematic illustrates the three main 447 interacting surfaces, highlighted in the right panel. (bcd) Detailed view of the molecular 448 interactions at each of the three interfaces, which close the HUWE $1_{N}$ ring. (e) Numerical 449 parameters of the three interfaces derived by PISA analysis. The average B-factor of the 450 HUWE1 $1_{N}$ crystal structure is $105 \AA^{2}$. 
452 Figure 5 - Solution behavior of HUWE1 $_{N}$. (a) XL-MS analysis of HUWE1 $1_{N}$. The highest 453 confidence detected cross-links are displayed on the primary structure of HUWE1 $1_{\mathrm{N}}$. Cross-links 454 between the neck and tail, supporting the closed ring structure, are shown in green, while 455 cross-links between the HECT domain and the solenoid ring are shown in orange and all other 456 cross-links in gray. (b) Sharpened electron density maps of the two CryoEM classes in two 457 different views showing different degrees of ring opening. Maps are colored according to the 458 schematic in Fig. 2a. (c) Breaking and shifting the ring-closure interfaces. The neck and tail 459 contacts are compared between the crystal structure (cyan) and two cryo-EM classes (domain460 based color code). The differences are summarized in a schematic cartoon (d). 
462 Figure 6 - Regulation of the HUWE1 $1_{N}$ HECT domain. (a) The HUWE1 $1_{N}$ active site is spatially 463 separated from the substrate binding ring. Catalytic cysteine and center of substrate binding 464 ring are highlighted in green. (b) Binding of E2-Ub to the HECT domain is not impeded in the 465 closed state of the full-length enzyme. The HUWE1 ${ }_{N}$ HECT domain (N-lobe, orange; C-lobe, 466 yellow) is superimposed with the crystal structure of the NEDD4L HECT domain (red) in complex 467 with Ub (blue)-charged UbcH5b (magenta) (PDB 3JVZ) (c) Steric clashes between the HUWE1 468 solenoid and a ubiquitin-charged HECT domain. The structure of the Rsp5 HECT domain (in red), 469 charged with Ub (in blue) and in complex with a peptide substrate (PDB 4LCD), is superimposed 470 on the HUWE1N HECT domain. Resultant clashes between the HECT C-lobe and ARM8-10 are

471 highlighted in the zoomed-in drawing. (d) The isolated HUWE1 $1_{N}$ HECT domain shows weak 472 substrate ubiquitination. Ubiquitination of DyLight488-labelled Rpl26 was visualized by in-gel 473 fluorescence. (e) Dysregulation of the isolated HECT domain. Autoubiquitination in the absence 474 of a substrate was followed by using DyLight488-labelled ubiquitin. The autoubiquitinated 475 products are indicated. (f) Single-turnover chase experiments of $1 \mu \mathrm{M}$ E2 loaded with 476 DyLight488-labelled ubiquitin by $2 \mu \mathrm{M} \mathrm{HUWE1}$ (Full-length and HECT), with or without $2 \mu \mathrm{M}$ 477 histone $\mathrm{H} 1$. 


\section{Methods}

480 Protein expression and purification

481 Expression of HUWE1 $\underline{\text { in insect cells }}$

482 The HUWE1 genes from Nematocida sp. ERTm5 and C. elegans were codon optimized for 483 expression in insect cells and synthesized in, respectively, three and five Bsal-flanked 484 fragments. Fragments were scarlessly assembled via the Bsal-GoldenGate reaction into a 485 pGBdest vector from the GoldenBac system ${ }^{41}$ together with a 3C-cleavable $\mathrm{N}$-terminal 486 hexahistidine tag, a Polyhedrin promoter and a SV40 terminator. The HUWE $1_{\mathrm{N}}-C A$ mutation 487 (C2457A), HUWE1 $1_{N}-I D R 1$ (1442-1522 replaced by GGSx6) and HUWE1 $\mathrm{N}^{-}$IDR2 (1610-1670 488 replaced by GGSx6) were generated by PCR-based site-directed mutagenesis prior to assembly.

489 Source plasmids containing the target constructs were transformed into DH10EMBacY cells.

490 Blue-white screening was used to isolate colonies containing recombinant baculoviral shuttle 491 vectors (bacmids). Bacmid DNA was extracted by alkaline lysis and isopropanol precipitation. 492 Bacmids were then transfected into adherent Spodoptera frugiperda (Sf9) insect cells in 6-well 493 plates, using Fugene HD transfection reagent (Promega). Successful transfection was tracked by 494 monitoring fluorescence of YFP, encoded by the bacmid backbone. All Sf9 insect cell culture 495 works were performed using ESF921 serum-free growth medium (Expression Systems) without 496 antibiotic supplementation. Following virus amplification in Sf9 suspension insect cell culture, 497 recombinant HUWE1 ${ }_{N}$ were expressed in Trichoplusia ni High-Five insect cells (Thermo Fisher) 498 infected at a density of $2 \times 10^{6} \mathrm{ml}^{-1}$ with the appropriate virus and grown at $21^{\circ} \mathrm{C}$ and 499120 r.p.m. using Insect Xpress Protein-free Insect Cell Medium (Lonza) supplemented with 
500 GlutaMAX (GIBCO) and Pen/Strep Amphotericin B (Lonza). Cells were harvested 4 days after

501 transfection by centrifugation at $4000 \times \mathrm{g}$ for 20 minutes, and pellets were flash-frozen in liquid

502 nitrogen and stored at $-80^{\circ} \mathrm{C}$. For generation of selenomethionine-substituted protein $\mathrm{Hi} 5$ cells

503 were grown in ESF 921 Delta Series medium (expressionsystems). Afterwards cells were

504 centrifuged and media exchanged to ESF 921 Delta Series Methionine Deficient medium

505 (expressionsystems). Cell suspension $\left(2 \times 10^{6}\right.$ cells per liter) was infected with baculovirus, and

506 swayed for $8 \mathrm{~h}$ at $27^{\circ} \mathrm{C}$. Then $100 \mathrm{mg} / \mathrm{L}$ Seleno-methionine were supplemented and cultures

507 swayed for 3 days at $21^{\circ} \mathrm{C}$ before harvesting.

508 Purification of HUWE1 $\underline{N}$

509 Purification procedures were carried out at $4^{\circ} \mathrm{C}$, using ÄKTA pure 25 (GE Healthcare)

510 instruments. Frozen cell pellet from $2 \mathrm{~L}$ of expression culture was rapidly thawed and

511 resuspended in $150 \mathrm{~mL}$ of Buffer $\mathrm{A}(50 \mathrm{mM}$ HEPES, $300 \mathrm{mM} \mathrm{NaCl}, 0.5 \mathrm{mM}$ TCEP, 20mM

512 imidazole, $\mathrm{pH}$ 7.5) with addition of four tablets of Complete EDTA-free Protease Inhibitor

513 (Roche) and $50 \mu \mathrm{L}$ Benzonase (IMP Molecular Biology Service), followed by centrifugation for 30

514 min at $40000 \times$ g.. After centrifugation, the soluble fraction was applied on a $5 \mathrm{~mL}$ HisTrap HP

515 column (GE Healthcare) pre-equilibrated with Buffer A. The column was washed with 10

516 column volumes (CV) with Buffer A, followed by 7 CV of 9\% Buffer B (50 mM HEPES, 300 mM

$517 \mathrm{NaCl}, 0.5 \mathrm{mM}$ TCEP, $300 \mathrm{mM}$ imidazole, $\mathrm{pH}$ 7.5) and $3 \mathrm{CV}$ of $12 \%$ Buffer $\mathrm{B}$, after which HUWE1 ${ }_{\mathrm{N}}$

518 was eluted in $100 \%$ Buffer B. Fractions containing HUWE1 $1_{N}$ were identified by SDS-PAGE and

519 dialyzed for 12 hours against Buffer C (50 mM HEPES, 150 mM NaCl 0.5 mM TCEP, pH 7.5). After

520 the dialysis protein was loaded onto a $6 \mathrm{~mL}$ Resource $\mathrm{Q}$ anion exchange column (GE Healthcare)

521 equilibrated with Buffer C. The column was washed for 3 CV with Buffer C, and then the protein 
522 was eluted with a linear 0-100\% gradient for 30 CV against Buffer D (50 mM HEPES, $1000 \mathrm{mM}$

$523 \mathrm{NaCl}, 0.5 \mathrm{mM}$ TCEP, $\mathrm{pH}$ 7.5). Pooled HUWE1 ${ }_{\mathrm{N}}$ fractions were concentrated to a final volume of 5

$524 \mathrm{~mL}$ using a Vivaspin 6 centrifugal concentrator (100 kDa cutoff, Sigma-Aldrich) and applied to a

525 Superose 6 Prep Grade HiLoad column (GE Healthcare) equilibrated with Buffer E (10 mM

$526 \mathrm{Na}_{2}\left(\mathrm{HPO}_{4}\right), 50 \mathrm{mM} \mathrm{NaCl}, 0.5 \mathrm{mM}$ TCEP, $\mathrm{pH}$ 7.2).

527 For purification of HUWE1 $1_{N}-C A$ for crystallization, the purification tag was cleaved with $3 C$

528 PreScission Protease (IMP Molecular Biology Service) after the first HisTrap step. Protein sample

529 was again applied on a $5 \mathrm{~mL}$ HisTrap HP column pre-equilibrated with Buffer A, untagged

530 protein was collected from the flow-through and loaded on $6 \mathrm{~mL}$ Resource $\mathrm{Q}$ anion exchange

531 column as described, followed by final step of size-exclusion chromatography. When HUWE1 $\mathrm{N}$

532 and variants were purified for assays, the reverse HisTrap step was performed in Buffer C, after

533 which the protein was concentrated and flash frozen. CeHUWE1 purification followed this

534 method, but with an additional SEC step.

535 The HUWE1 $1_{N}$ HECT domain was cloned into a pET28 vector with a PreScission cleavable N-

536 terminal $\mathrm{His}_{6}$ tag, and expressed in BL21 cells with IPTG induction at $18^{\circ} \mathrm{C}$. Cells were harvested

537 by sonication and purified using a $5 \mathrm{~mL}$ HisTrap (GE Healthcare) in buffer containing $50 \mathrm{mM}$

538 HEPES, $500 \mathrm{mM} \mathrm{NaCl}, \mathrm{pH}$ 7.4, before size-exclusion chromatography using a HiLoad 16/60

539 Superdex 200 column (GE Healthcare) equilibrated in $50 \mathrm{mM}$ Tris- $\mathrm{HCl}, 200 \mathrm{mM} \mathrm{NaCl}, \mathrm{pH}$ 7.4.

540 Ribosome preparation

541 HEK293T cells in DMEM medium were incubated with $10 \mathrm{ng} / \mathrm{mL}$ cycloheximide and lysed with $5421 \%$ Triton X-100 and $1 \%$ sodium deoxycholate. The cleared lysate was applied to $6 \times 2 \mathrm{~mL} 30 \%$ 
543 Sucrose Cushion (10mM Tris pH 7.5, $100 \mathrm{mM} \mathrm{NaCl}, 10 \mathrm{mM} \mathrm{MgCl}$ ) and centrifuged at $116000 \mathrm{x}$

$544 \mathrm{~g}$ at $4^{\circ} \mathrm{C}$ for 5 hours. The pellets were washed with_50mM Tris, $10 \mathrm{mM} \mathrm{NaCl}, 10 \mathrm{mM} \mathrm{MgCl} 2,100$

$545 \mathrm{mM} \mathrm{NH}_{4} \mathrm{Cl} \mathrm{pH} 8$ and resuspended in $30 \mathrm{mM}$ Tris, $100 \mathrm{mM} \mathrm{NaCl}, 10 \mathrm{mM} \mathrm{MgCl}$. Insoluble

546 contaminants were removed by centrifugation. The soluble material was then applied to a 12

$547 \mathrm{~mL} 15-30 \%$ sucrose gradient in $20 \mathrm{mM}$ Tris $\mathrm{pH} 7.5,100 \mathrm{mM} \mathrm{NaCl}, 10 \mathrm{mM} \mathrm{MgCl}$. Gradients were

548 centrifuged for $100 \mathrm{~min}$ at $4^{\circ} \mathrm{C}$ and $100000 \mathrm{xg}$ and fractions containing ribosomes were pooled

549 and concentrated.

550 Reagents for ubiquitination assays

551 Pure human Histone $\mathrm{H} 1$, Histone $\mathrm{H} 2 \mathrm{~B}$ and native nucleosomes were purchased from Sigma-

552 Aldrich. 4WJ DNA was generated by mixing equimolar oligonucleotide substrates (Sigma-

553 Aldrich): 5'GAATTCAGCACGAGTCCTAACGCCAGATCT,

554 5'AGATCTGGCGTTAGgTGATACCGATGCATC， 5'GATGCATCGGTATCAGGCTTACGACTAGTG， and

555 5'CACTAGTCGTAAGCCACTCGTGCTGAATTC at $100 \mu \mathrm{M}$ in buffer containing $10 \mathrm{mM}$ Tris- $\mathrm{HCl}, 100$

$556 \mathrm{mM} \mathrm{NaCl}, \mathrm{pH} 8.0$, incubating at $95^{\circ} \mathrm{C}$ for two minutes and then slowly cooling.

557 Human Mcl-1, amino acids 2-330 was produced as a GST fusion protein in E. coli, using strain

558 BL21(DE3) Al. Cells were grown in TB media, induced with $0.2 \%$ arabinose, and grown overnight

559 at $22^{\circ} \mathrm{C}$. Cells were lysed by sonication in $50 \mathrm{mM}$ Tris buffer at $\mathrm{pH} 7.5$, containing $200 \mathrm{mM} \mathrm{NaCl}$

560 and $5 \mathrm{mM} \mathrm{DTT}$. After centrifugation to remove cell debris, the protein was bound to glutathione

561 agarose and was eluted in the same buffer containing $25 \mathrm{mM}$ reduced glutathione. The GST tag

562 was removed by overnight cleavage with TEV protease followed by reverse affinity after

563 desalting into buffer without glutathione. Untagged Mcl-1 was further purified by separation on 
564 a MonoQ ion exchange column, in $50 \mathrm{mM}$ Tris buffer at pH 7.8 containing $1 \mathrm{mM}$ TCEP, using a 565 gradient from $50 \mathrm{mM}$ to $1 \mathrm{M} \mathrm{NaCl}$ over 5 column volumes. $\mathrm{Mcl}-1$ eluted at $220 \mathrm{mM} \mathrm{NaCl}$, was 566 concentrated to $13 \mathrm{mg} / \mathrm{mL}$ and stored at $-80^{\circ} \mathrm{C}$.

567 The forkhead domain of human FoxP3, amino acids 336 to 419, was expressed as a His-tagged 568 protein in E. coli strain BL21(DE3) in autoinduction media at 18 degrees C. Cells were lysed by 569 sonication in $25 \mathrm{mM}$ Tris buffer at pH 7.8 containing $200 \mathrm{mM} \mathrm{NaCl}, 1 \mathrm{mM}$ TCEP, $50 \mathrm{mM}$ 570 arginine, $0.5 \%$ glycerol, and $0.02 \%$ CHAPS. After centrifugation to remove cell debris, the

571 protein was bound to Nickel beads, the beads were washed with $20 \mathrm{mM}$ Tris buffer at pH 8 572 containing $200 \mathrm{mM} \mathrm{NaCl}, 1 \mathrm{mM}$ TCEP, $50 \mathrm{mM}$ arginine, $0.5 \%$ glycerol, and eluted in the same 573 buffer plus $500 \mathrm{mM}$ imidazole. The protein was incubated overnight with TEV protease to 574 remove the His tag and simultaneously dialyzed into $20 \mathrm{mM}$ Tris buffer at $\mathrm{pH} 8.0$ containing 200 $575 \mathrm{mM} \mathrm{NaCl}$ and $1 \mathrm{mM}$ TCEP. After dialysis the protein was concentrated and further purified over 576 a Superdex 75 size exclusion column equilibrated in $25 \mathrm{mM}$ Tris buffer at pH 7.5 containing 150 $577 \mathrm{mM} \mathrm{NaCl}$ and $1 \mathrm{mM} \mathrm{TCEP}$. The protein was concentrated to $4.7 \mathrm{mg} / \mathrm{mL}$ and stored at $-80^{\circ} \mathrm{C}$.

578 Recombinant human UBA1 was provided by the Vienna BioCenter core facilities. Ubiquitin was 579 expressed in BL21(DE3) cells using autoinduction media. Cells were lysed by sonication in 50 $580 \mathrm{mM}$ ammonium acetate $\mathrm{pH} 4.5$, and the cleared lysate was subjected to heat treatment at $70^{\circ} \mathrm{C}$ 581 to remove impurities. Ubiquitin was then further purified with a SP Sepharose Fast Flow ion582 exchange column (GE Healthcare) followed by SEC using a Superdex 75 Increase column (GE 583 Healthcare).

584 The Rpl-26 and Let-70 (UbcH5b) genes from C. elegans were generated as double-stranded DNA 585 fragments (IDT) and cloned into a modified pET29b expression vector (containing an $\mathrm{N}$-terminal 
586 His6-tag and TEV cleavage site) using NEB HiFi assembly (New England Biolabs). The C. elegans

587 HECT domain (3793-4180) vector was constructed similarly. Expression was performed by IPTG

588 induction in E. coli BL21 (DE3) cells at $20^{\circ} \mathrm{C}$ overnight. Cell pellets were resuspended in lysis

589 buffer ( $50 \mathrm{mM}$ Tris, $150 \mathrm{mM} \mathrm{NaCl}, 10 \mathrm{mM}$ imidazole, Complete EDTA-free protease inhibitor

590 cocktail at $\mathrm{pH}$ 7.5) before sonication to induce cell lysis. Cell lysates were clarified through

591 centrifugation at $18,500 \mathrm{~g}$ for 20 minutes at $4^{\circ} \mathrm{C}$. Clarified lysates were then incubated with $\mathrm{Ni}$ -

592 NTA resin (Qiagen) for 1 hour at $4^{\circ} \mathrm{C}$ with mild agitation. Ni-NTA resin was washed before

593 elution with $150 \mathrm{mM}$ imidazole. Proteins were further purified by SEC using a Superdex 75

594 16/600 column (GE Healthcare) into $50 \mathrm{mM}$ Tris, $150 \mathrm{mM} \mathrm{NaCl}, 0.5 \mathrm{mM}$ TCEP at pH 7.5. Rpl-26

595 or ubiquitin bearing a single $\mathrm{N}$-terminal cysteine residue were buffer exchanged into degassed

596 labelling buffer ( $50 \mathrm{mM}$ Tris, $150 \mathrm{mM} \mathrm{NaCl}$ at $\mathrm{pH}$ 7), before addition of a 2:1 molar excess of

597 maleimide fluorophore (Dylight488 or Dylight800, Thermo Fischer Scientific). Reactions were

598 flushed with argon and incubated in the dark for 2 hours at room temperature. Reactions were

599 stopped by desalting to remove excess fluorophore using Centri Pure P2 columns.

\section{Ubiquitination assays}

601 Ubiquitination reactions were performed in buffer containing $50 \mathrm{mM}$ HEPES pH 7.5, $150 \mathrm{mM}$

$602 \mathrm{NaCl}, 5 \mathrm{mM} \mathrm{MgCl}_{2}$ and $0.05 \mathrm{mM}$ TCEP. Reactions contained $0.5 \mu \mathrm{M}$ Uba1, $2.5 \mu \mathrm{M} \mathrm{CeUbcH5b}, 10$

$603 \mu \mathrm{M}$ ubiquitin and $0.5 \mu \mathrm{M}$ HUWE1 $1_{N}$. Substrates were usually added at $4 \mu \mathrm{M}$ concentration. For

604 fluorescent visualization of ubiquitinated products, reactions were spiked with $0.5 \mu \mathrm{M} U \mathrm{~b}$ -

605 Dylight488. For the comparative reactions of Rpl26 and ribosomes, or histone H2B and 606 nucleosomes, only $0.5 \mu \mathrm{M}$ substrate was used, and $2 \mu \mathrm{M}$ labelled ubiquitin (Ub-DyLight488 for 
607 the nucleosomes, Ub-DyLight800 for the ribosomes) to increase sensitivity. To compare the

608 ubiquitination of Rpl26 by the HECT domain and full-length HUWE $1_{N}$, labelled substrate, Rpl26-

609 DyLight488, was employed to avoid overlapping autoubiquitination bands. All reactions were

610 initiated by addition of $5 \mathrm{mM} \mathrm{ATP}$ and incubated at $30^{\circ} \mathrm{C}$ for the indicated time. Samples were

611 quenched by addition of 2x reducing SDS gel loading buffer and run on 4-12\% NuPAGE Bis-Tris

612 gradient gels (Invitrogen) in MES running buffer. Fluorescence imaging was performed using a

613 ChemiDoc MP system (Bio-Rad), and then gels were coomassie stained to visualize total protein

614 content. CeHUWE1 reactions used the same method, but with $2 \mu \mathrm{M}$ substrate and $0.5 \mu \mathrm{M}$ Ub-

615 Dylight800. For single turnover experiments, $0.5 \mu \mathrm{M}$ Uba1, $8 \mu \mathrm{M}$ CeUbcH5b, $10 \mu \mathrm{M}$ ubiquitin

616 and $0.5 \mu \mathrm{M}$ ubiquitin-DyLight488 were incubated at $37^{\circ} \mathrm{C}$ in the usual reaction buffer but with 3

$617 \mathrm{mM} \mathrm{MgCl}_{2}$ and $3 \mathrm{mM}$ ATP. The reactions were then quenched with $20 \mathrm{mM}$ EDTA on ice and

618 diluted 1 in 8 (final E2 concentration of $1 \mu \mathrm{M}$ ) in reactions containing $2 \mu \mathrm{M}$ HUWE1 $1_{N}$ or HECT

619 domain with or without $2 \mu \mathrm{M}$ histone $\mathrm{H} 1$, in the normal reaction buffer but without $\mathrm{MgCl}_{2}$, and

620 then incubated at $30^{\circ} \mathrm{C}$ for the indicated time points before quenching with either reducing or

621 non-reducing 2x SDS gel loading buffer. Technical replicates of all biochemical assays were

622 performed.

\section{Crystallization and tantalum bromide soaking of HUWE1 $1_{\mathrm{N}}-\mathrm{CA}$}

$624 \mathrm{HUWE} 1_{\mathrm{N}}-\mathrm{CA}$ crystals were obtained using the sitting drop vapor diffusion method at 277K. 1.5

$625 \mu \mathrm{L}$ protein solution $(5.1 \mathrm{mg} / \mathrm{mL})$ was mixed with $0.75 \mu \mathrm{L}$ reservoir solution containing $10.4 \%$

$626(\mathrm{w} / \mathrm{v})$ PEG 4000, $100 \mathrm{mM} \mathrm{MgCl} 2,100 \mathrm{mM}$ HEPES pH 6.6 and $6 \mathrm{mM}$ DTT. The crystals were

627 cryoprotected with $23 \%$ ethylene glycolbefore flash freezing. Crystals of selenomethionine- 
628 substituted $\mathrm{HUWE} 1_{\mathrm{N}}-\mathrm{CA}$ were obtained in the same condition with a protein concentration of $6295.6 \mathrm{mg} / \mathrm{mL}$.

630 To obtain a heavy-atom derivative for experimental phasing, crystals were soaked with

631 hexatantalum tetradecabromide from the Tantalum Cluster Derivatization kit (Jena

632 Biosciences). $1.8 \mu \mathrm{L}$ of mother liquor supplemented with hexatantalum tetradecabromide were

633 mixed with drops containing crystals and incubated for 1 hour before crystals were flash-frozen

634 in liquid nitrogen.

\section{HUWE1 $_{\mathrm{N}}-\mathrm{CA}$ structure solution and refinement}

636 Diffraction data was collected at X06SA at the Swiss Light Source at a wavelength of $1 \AA$. The

637 data were autoprocessed in P1 using autoPROC ${ }^{42}$, implementing XDS ${ }^{43}$, the STARANISO server ${ }^{44}$

638 and Aimless ${ }^{45}$ for data integration, scaling and merging. Two copies of the human HUWE1 HECT

639 domain (PDB 3G1N) were placed by molecular replacement using PHASER ${ }^{46}$, however did not

640 allow to build the huge $\mathrm{N}$-terminal part into the electron density map. For experimental

641 phasing, we used anomalous diffraction data from $\mathrm{Ta}_{6} \mathrm{Br}_{14}$ soaked crystals. Tantalum sites were

642 localized and refined with PHENIX AutoSol ${ }^{47}$. The initial model, which comprised the HECT

643 domain and a poly-alanine scaffold of the ARM solenoid, was manually built. Further rounds of

644 modeling and refinement were performed with $\operatorname{Coot}^{48}$ and BUSTER ${ }^{49}$ using NCS restraints and

645 TLS. The sequence was placed with the aid of an anomalous map calculated from the SeMet

646 dataset to $5 \AA$ resolution using BUSTER and co-evolutionary contact prediction by the RaptorX

647 sever $^{31}$. Specifically, for small canonical ARM repeat stretches, the predicted RaptorX model

648 was docked as an initial structure. For the rest of the protein, predicted contacts were used 
649 alongside bulky residue density and anomalous SeMet signal to initially assign the register of

650 helices that were next to helices of known sequence. Additionally, the regions outside of the

651 solenoid, i.e. Ins1-4, could be readily identified due to their distinct contact pattern, preventing

652 large errors in initial sequence assignment. The final structure had $R_{\text {work }} / R_{\text {free }}$ values of

$65320.24 / 23.30 \%$ and $0.21 \%$ Ramachandran outliers and 94.7\% favored. Chain A was used for all

654 structural figures, made with PyMOL.

\section{Negative stain EM of CeHUWE1}

656 Copper/palladium grids (Agar Scientific) were covered with a $4 \mathrm{~nm}$ carbon-layer, performed in-

657 house. Glow-discharge of grids was performed using a BAL-TEC sputter coater CD005 for 120

658 seconds. CeHUWE1 was diluted to $0.02 \mathrm{mg} / \mathrm{ml}$ in $10 \mathrm{mM} \mathrm{HEPES}, 100 \mathrm{mM} \mathrm{NaCl} \mathrm{pH} 7.5$ and 659 applied to the grid for 60 seconds prior to staining with $2 \%$ uranyl acetate. A 200kV FEI Tecnai 660 G2 20 (T20) transmission electron microscope was used to image CeHUWE1 grids at a 50,000x 661 magnification (pixel size $2.211 \AA$ A). The micrographs obtained were processed using Relion $3.1^{50}$. 662 Initial number of particles following auto-picking was $\sim 65,000$, reduced to $\sim 14,000$ at the final $6632 \mathrm{D}$ classification, and $\sim 9,000$ for the final 3D model.

\section{Cryo-EM sample preparation and data collection}

$6654 \mu \mathrm{L}$ of $\mathrm{HUWE}_{\mathrm{N}}$ at $0.8 \mathrm{mg} / \mathrm{mL}$ were applied to a Quantifoil R2/2 Cu 200 mesh grid, freshly glow666 discharged at $20 \mathrm{~mA}$ for $60 \mathrm{~s}$ in a SCD 005 Sputter Coater (BAL-TEC). After $1 \mathrm{~s}$ pre-blotting time, 667 the sample was blotted for $2 \mathrm{~s}$ with a relative humidity of $70 \%$ and a temperature of $4^{\circ} \mathrm{C}$ in an 668 EM GP freeze plunger (Leica Microsystems). Cryo-EM grids were screened using a Glacios 
669 equipped with a Falcon3EC camera (Thermo Fisher Scientific). The selected grid was recorded

670 on a Titan Krios electron microscope (ThermoFisher) equipped with a K3 camera (Gatan) in

671 counting mode at the Institute of Science and Technology Austria. The dataset was recorded

672 both untilted and at $25^{\circ}$ stage tilt. Micrographs were collected with EPU for the untilted and

673 SerialEM ${ }^{51}$ for the tilted part of the dataset. The microscope was operated in nanoprobe mode

674 with a beam diameter of $1200 \mathrm{~nm}$ at a nominal magnification of $105000 \mathrm{x}$, resulting in a

675 calibrated pixel size of $0.86 \AA ̊$ per pixel. Images were collected in super-resolution. Four images

676 were taken per hole from different non-overlapping areas using image beam shift. The total

677 electron dose of 60 electrons per $\AA^{2}$ was fractionated into 40 frames. A total number of 2580

678 movies were acquired without tilt, and 1958 movies were acquired at tilt. A defocus range from

$679-2$ to $-3.5 \mu \mathrm{m}$ was used.

\section{Cryo-EM image processing and model refinement}

681 Processing was performed in Relion $3 \cdot 1.0^{50}$. Movie frames were motion-corrected, dose-

682 weighted and super-resolution images were binned two-fold using MotionCor2 ${ }^{52}$. Tilted and

683 non-tilted micrographs were merged afterwards (4538 micrographs). Micrographs with strong

684 drift were discarded. The Contrast transfer function (CTF) parameters were determined using

685 CTFFIND4.1.13 $3^{53}$. Only the micrographs in which Thon rings extended to $5 \AA$ in the untilted or to

$6867 \AA$ in tilted dataset were included in the further processing steps. This yielded 1847 good

687 micrographs for the non-tilted and 919 for the tilted part of the dataset. Particles were picked

688 using $\mathrm{CrYOLO}^{54}$. As a first step, particles from 8 micrographs were picked manually in order to

689 train the neural network. After the training, particles were picked using the threshold of 0.3 and 
690 extracted at $2 \times$ pixel size. Subsequently, several iterations of $2 \mathrm{D}$ classification were performed, 691 using subsets with maximum 200000 particles. After this procedure, the best class averages 692 were used to generate an initial 3D model. Afterwards 3D classification of all picked particles

693 with a 3D auto-refined map as initial reference, filtered to $30 \AA$. . This resulted in two good 694 classes. CTF parameters and per-particle trajectories were then refined in an iterative manner, 695 one round of CTF refinement was followed by Bayesian polishing and another CTF refinement. 696 This led to a refined HUWE1 $1_{N}$ map of $6.49 \AA$ resolution. Particles were further 3D classified 697 without searches and with increased $T$ value to remove damaged particles and sort the 698 heterogeneity. This resulted in two classes, corresponding to a closed (Class 1 ) and open (Class 699 2) state, with an overall resolution, as judged by an FSC of 0.143 , of $5.3 \AA$ and $6.4 \AA$ respectively. 700 Heterogeneity analysis was performed with CryoDRGN ${ }^{55}$.

701 For modelling, Class 1 and Class 2 were sharpened with a B factors of $-137 \AA^{2}$ and $-178 \AA^{2}$ 702 respectively. Our HUWE1 $1_{N}$ crystal structure was docked onto the maps using UCSF Chimera ${ }^{56}$. 703 The structure was then manually modeled in $\mathrm{Coot}^{48}$ mostly through rigid body fitting of sections 704 of the structure. Real space refinement was performed using Phenix ${ }^{57}$.

\section{Mass spectrometry analysis}

706 To determine ubiquitination linkage types, a HUWE1 $1_{N}$ autoubiquitination reaction was 707 performed overnight and separated by SDS-PAGE. To determine Rpl26 ubiquitination sites, the 708 reaction was performed for two hours. The ubiquitinated products were excised and then 709 digested with trypsin. NanoLC-MS analysis was performed using an UltiMate 3000 RSLC nano 710 system (Thermo Fisher Scientific) coupled to a Q Exactive HF-X mass spectrometer (Thermo 
711 Fisher Scientific), equipped with a Proxeon nanospray source (Thermo Fisher Scientific).

712 Peptides were loaded onto a PepMap C18 trap column (Thermo Fisher Scientific) at a flow rate

713 of $25 \mu \mathrm{L} \mathrm{min}{ }^{-1}$ using $0.1 \%$ TFA as mobile phase. After $10 \mathrm{~min}$, the trap column was switched in

714 line with a PepMap C18 analytical column (Thermo Fisher Scientific). Peptides were eluted

715 using a binary 2 hour gradient, starting with 98\% A (water/formic acid, 99.9/0.1, v/v) and 2\% B

716 (water/acetonitrile/formic acid, 19.92/80/0.08, v/v/v), increasing to $35 \%$ B over $180 \mathrm{~min}$, to

$71790 \%$ B over 5 min before decreasing back to $98 \%$ A and $2 \%$ B.

718 The mass spectrometer was operated in data-dependent mode, using a full scan (m/z range

$719350-1600)$ followed by $15 \mathrm{MS} / \mathrm{MS}$ scans of the 15 most abundant ions. MS/MS spectra were

720 acquired using normalized collision energy of $26,28,30$, isolation width of $1.0 \mathrm{~m} / \mathrm{z}$, resolution

721 of 30,000 and the target value was set to 50,000 . Precursor ions of charge state +3 to +7 were

722 fragmented.

723 For peptide identification, the RAW-files were loaded into Proteome Discoverer (version

724 2.3.0.523, Thermo Scientific). All MS/MS spectra were searched using MSAmanda

$725 \mathrm{v} 2.0 .0 .9849^{58}$. The peptide mass tolerance was set to $\pm 5 \mathrm{ppm}$ and the fragment mass tolerance

726 to $15 \mathrm{ppm}$. The maximal number of missed cleavages was set to 2, using tryptic enzymatic

727 specificity. The result was filtered to 1\% FDR on protein level in Thermo Proteome Discoverer.

728 This sub-database was then searched again for all standard amino acid modifications. Peptide

729 areas were quantified using in-house-developed tool apQuant ${ }^{59}$.

730 Crosslinking for XL-MS analysis was performed with 0.06 mM DSS (Creative Molecule) for 30

$731 \mathrm{~min}$ at $20^{\circ} \mathrm{C}$, and quenched with ammonium bicarbonate. The sample was carboxymethylated 
732 in urea and then trypsin-digested overnight. Trypsin was deactivated with $0.4 \%$ TFA, before

733 loading on Sep-Pak cartridges (Fischer Chemicals) equilibrated in $5 \%$ acetonitrile, $0.1 \%$ formic

734 acid, and eluted with 50\% acetonitrile. SEC in 30\% acetonitrile, $0.1 \%(\mathrm{v} / \mathrm{v})$ TFA was performed

735 using a Superdex 30 Increase 3.2/300 column (GE Healthcare) to enrich for crosslinked

736 peptides. NanoLS-MS analysis was performed as above, but with a 3 hour HPLC gradient.

737 Identified crosslinks were then filtered to 0.1\% FDR on link level and visualized with XiView ${ }^{60}$.

738 Only one self-link was observed. A technical replicate was performed.

739 


\section{References}

741 1. Ciechanover, A., Orian, A. \& Schwartz, A.L. Ubiquitin-mediated proteolysis: biological 742 regulation via destruction. Bioessays 22, 442-51 (2000).

$7432 . \quad$ Hochstrasser, M. Lingering mysteries of ubiquitin-chain assembly. Cell 124, 27-34 (2006).

744 3. Cardozo, T. \& Pagano, M. The SCF ubiquitin ligase: insights into a molecular machine. $745 \quad$ Nat Rev Mol Cell Biol 5, 739-51 (2004).

746 4. Alfieri, C., Zhang, S. \& Barford, D. Visualizing the complex functions and mechanisms of 747 the anaphase promoting complex/cyclosome (APC/C). Open Biol 7(2017).

748 5. Kamadurai, H.B. et al. Mechanism of ubiquitin ligation and lysine prioritization by a HECT $749 \quad$ E3. Elife 2, e00828 (2013).

750 6. Chen, Z. et al. A Tunable Brake for HECT Ubiquitin Ligases. Mol Cell 66, 345-357.e6 $751 \quad$ (2017).

752 7. Lorenz, S. Structural mechanisms of HECT-type ubiquitin ligases. Biol Chem 399, 127-145 753 (2018).

754 8. Kao, S.H., Wu, H.T. \& Wu, K.J. Ubiquitination by HUWE1 in tumorigenesis and beyond. J $755 \quad$ Biomed Sci 25, 67 (2018).

756 9. Zhong, Q., Gao, W., Du, F. \& Wang, X. Mule/ARF-BP1, a BH3-only E3 ubiquitin ligase, 757 catalyzes the polyubiquitination of $\mathrm{Mcl}-1$ and regulates apoptosis. Cell 121, 1085-95 $758 \quad$ (2005).

759 10. Inoue, S. et al. Mule/Huwe1/Arf-BP1 suppresses Ras-driven tumorigenesis by preventing 760 c-Myc/Miz1-mediated down-regulation of p21 and p15. Genes Dev 27, 1101-14 (2013). 
761 11. Chen, D. et al. ARF-BP1/Mule is a critical mediator of the ARF tumor suppressor. Cell $762 \quad 121,1071-83(2005)$.

763 12. Adhikary, S. et al. The ubiquitin ligase HectH9 regulates transcriptional activation by Myc 764 and is essential for tumor cell proliferation. Cell 123, 409-21 (2005).

765 13. Urbán, N. et al. Return to quiescence of mouse neural stem cells by degradation of a 766 proactivation protein. Science 353, 292-5 (2016).

767 14. Hao, Z. et al. K48-linked KLF4 ubiquitination by E3 ligase Mule controls T-cell 768 proliferation and cell cycle progression. Nat Commun 8, 14003 (2017).

769 15. DeGroot, R.E.A. et al. Huwe1-Mediated Ubiquitylation of Dishevelled Defines a Negative $770 \quad$ Feedback Loop in the Wnt Signaling Pathway. Science Signalling 7, ra26 (2014).

771 16. Hall, J.R. et al. Cdc6 stability is regulated by the Huwe1 ubiquitin ligase after DNA 772 damage. Mol Biol Cell 18, 3340-50 (2007).

773 17. Parsons, J.L. et al. Ubiquitin ligase ARF-BP1/Mule modulates base excision repair. EMBO $774 \quad$ J 28, 3207-15 (2009).

775 18. Wang, X. et al. HUWE1 interacts with BRCA1 and promotes its degradation in the 776 ubiquitin-proteasome pathway. Biochem Biophys Res Commun 444, 549-54 (2014).

777 19. Xu, Y., Anderson, D.E. \& Ye, Y. The HECT domain ubiquitin ligase HUWE1 targets 778 unassembled soluble proteins for degradation. Cell Discov 2, 16040 (2016).

779 20. Sung, M.K. et al. A conserved quality-control pathway that mediates degradation of $780 \quad$ unassembled ribosomal proteins. Elife 5(2016).

781 21. Liu, Z., Oughtred, R. \& Wing, S.S. Characterization of E3Histone, a novel testis ubiquitin 782 protein ligase which ubiquitinates histones. Mol Cell Biol 25, 2819-31 (2005). 
783 22. Singh, R.K., Kabbaj, M.H., Paik, J. \& Gunjan, A. Histone levels are regulated by phosphorylation and ubiquitylation-dependent proteolysis. Nat Cell Biol 11, 925-33 (2009).

786 23. Deshaies, R.J. Proteotoxic crisis, the ubiquitin-proteasome system, and cancer therapy. BMC Biol 12, 94 (2014).

788 24. Oromendia, A.B. \& Amon, A. Aneuploidy: implications for protein homeostasis and 789 disease. Dis Model Mech 7, 15-20 (2014).

790 25. Myant, K.B. et al. HUWE1 is a critical colonic tumour suppressor gene that prevents MYC 791 signalling, DNA damage accumulation and tumour initiation. EMBO Mol Med 9, 181-197 (2017).

793 26. Peter, S. et al. Tumor cell-specific inhibition of MYC function using small molecule 794 inhibitors of the HUWE1 ubiquitin ligase. EMBO Mol Med 6, 1525-41 (2014).

795 27. Froyen, G. et al. Submicroscopic duplications of the hydroxysteroid dehydrogenase HSD17B10 and the E3 ubiquitin ligase HUWE1 are associated with mental retardation. Am J Hum Genet 82, 432-43 (2008).

798 28. Jäckl, M. et al. $\beta$-Sheet Augmentation Is a Conserved Mechanism of Priming HECT E3 Ligases for Ubiquitin Ligation. J Mol Biol 430, 3218-3233 (2018).

29. Michel, M.A., Swatek, K.N., Hospenthal, M.K. \& Komander, D. Ubiquitin Linkage-Specific 801 Affimers Reveal Insights into K6-Linked Ubiquitin Signaling. Mol Cell 68, 233-246.e5 (2017).

803 30. White, A.E., Hieb, A.R. \& Luger, K. A quantitative investigation of linker histone 804 interactions with nucleosomes and chromatin. Sci Rep 6, 19122 (2016). 
805 31. Wang, S., Sun, S., Li, Z., Zhang, R. \& Xu, J. Accurate De Novo Prediction of Protein Contact Map by Ultra-Deep Learning Model. PLoS Comput Biol 13, e1005324 (2017).

807 32. Chen, V.B. et al. MolProbity: all-atom structure validation for macromolecular crystallography. Acta Crystallogr D Biol Crystallogr 66, 12-21 (2010).

33. Sander, B., Xu, W., Eilers, M., Popov, N. \& Lorenz, S. A conformational switch regulates the ubiquitin ligase HUWE1. Elife 6(2017).

811 34. Hunkeler, M. et al. Modular HUWE1 architecture serves as hub for degradation of cellfate decision factors. bioRxiv, 2020.08.19.257352 (2020).

813 35. Conti, E., Uy, M., Leighton, L., Blobel, G. \& Kuriyan, J. Crystallographic analysis of the

36. Huber, A.H. \& Weis, W.I. The structure of the beta-catenin/E-cadherin complex and the molecular basis of diverse ligand recognition by beta-catenin. Cell 105, 391-402 (2001).

818 37. Pandya, R.K., Partridge, J.R., Love, K.R., Schwartz, T.U. \& Ploegh, H.L. A structural element within the HUWE1 HECT domain modulates self-ubiquitination and substrate

821 38. Kamadurai, H.B. et al. Insights into ubiquitin transfer cascades from a structure of a UbcH5B approximately ubiquitin-HECT(NEDD4L) complex. Mol Cell 36, 1095-102 (2009).

823 39. Forget, A. et al. Shh signaling protects Atoh1 from degradation mediated by the E3 ubiquitin ligase Huwe1 in neural precursors. Dev Cell 29, 649-61 (2014).

825 40. Baek, K. et al. NEDD8 nucleates a multivalent cullin-RING-UBE2D ubiquitin ligation 826 assembly. Nature 578, 461-466 (2020). 
827 41. Neuhold, J. et al. GoldenBac: a simple, highly efficient, and widely applicable system for construction of multi-gene expression vectors for use with the baculovirus expression

42. Vonrhein, C. et al. Data processing and analysis with the autoPROC toolbox. Acta Crystallogr D Biol Crystallogr 67, 293-302 (2011).

832 43. Kabsch, W. XDS. Acta Crystallogr D Biol Crystallogr 66, 125-32 (2010).

833 44. Tickle, I.J., Flensburg, C., Keller, P., Paciorek, W., Sharff, A., Vonrhein, C., Bricogne, G. STARANISO. (Cambridge, United Kingdom: Global Phasing Ltd., 2018).

835 45. Evans, P.R. \& Murshudov, G.N. How good are my data and what is the resolution? Acta 836 Crystallogr D Biol Crystallogr 69, 1204-14 (2013).

837 46. McCoy, A.J. et al. Phaser crystallographic software. J Appl Crystallogr 40, 658-674 838 (2007).

839 47. Terwilliger, T.C. et al. Decision-making in structure solution using Bayesian estimates of map quality: the PHENIX AutoSol wizard. Acta Crystallogr D Biol Crystallogr 65, 582-601

842 48. Emsley, P., Lohkamp, B., Scott, W.G. \& Cowtan, K. Features and development of Coot. Acta Crystallogr D Biol Crystallogr 66, 486-501 (2010).

49. Smart, O.S. et al. Exploiting structure similarity in refinement: automated NCS and target-structure restraints in BUSTER. Acta Crystallogr D Biol Crystallogr 68, 368-80 (2012).

847 50. Zivanov, J. et al. New tools for automated high-resolution cryo-EM structure 848 determination in RELION-3. Elife 7(2018). 
849 51. Schorb, M., Haberbosch, I., Hagen, W.J.H., Schwab, Y. \& Mastronarde, D.N. Software 850 tools for automated transmission electron microscopy. Nat Methods 16, 471-477 (2019).

851 52. Zheng, S.Q. et al. MotionCor2: anisotropic correction of beam-induced motion for 852 improved cryo-electron microscopy. Nat Methods 14, 331-332 (2017).

853 53. Rohou, A. \& Grigorieff, N. CTFFIND4: Fast and accurate defocus estimation from 854 electron micrographs. J Struct Biol 192, 216-21 (2015).

855 54. Wagner, T. et al. SPHIRE-crYOLO is a fast and accurate fully automated particle picker for cryo-EM. Commun Biol 2, 218 (2019).

857 55. Zhong, E.D., Bepler, T., Berger, B. \& Davis, J.H. CryoDRGN: reconstruction of heterogeneous cryo-EM structures using neural networks. Nat Methods 18, 176-185 (2021).

56. Pettersen, E.F. et al. UCSF Chimera--a visualization system for exploratory research and analysis. J Comput Chem 25, 1605-12 (2004).

862 57. Adams, P.D. et al. PHENIX: a comprehensive Python-based system for macromolecular structure solution. Acta Crystallogr D Biol Crystallogr 66, 213-21 (2010).

864 58. Dorfer, V. et al. MS Amanda, a universal identification algorithm optimized for high accuracy tandem mass spectra. J Proteome Res 13, 3679-84 (2014).

866 59. Doblmann, J. et al. apQuant: Accurate Label-Free Quantification by Quality Filtering. J 867 Proteome Res 18, 535-541 (2019).

868 60. Graham, M., Combe, C., Kolbowski, L. \& Rappsilber, J. xiView: A common platform for 869 the downstream analysis of Crosslinking Mass Spectrometry data. bioRxiv, 561829 870 (2019). 
871

872 


\section{Figure 1}

a NTD - substrate targeting, regulation HECT UBAUIM WWE BH3 UBM

D $\begin{array}{lll}\text { UBM } & \text { CeHUWE1 } \\ \text { HUWE1 }\end{array}$

p53, Mcl-1, Miz1, Dsh, c-Myc, Cdc6
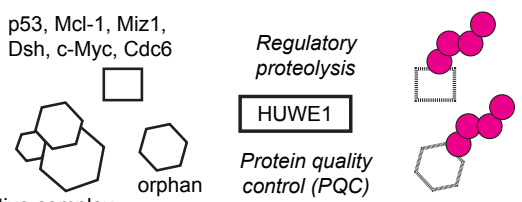

native complex protein

b Substrate screen HUWE1N

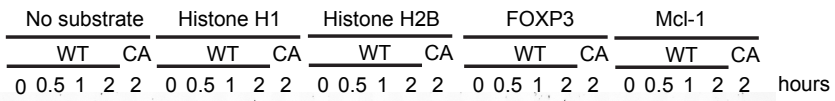

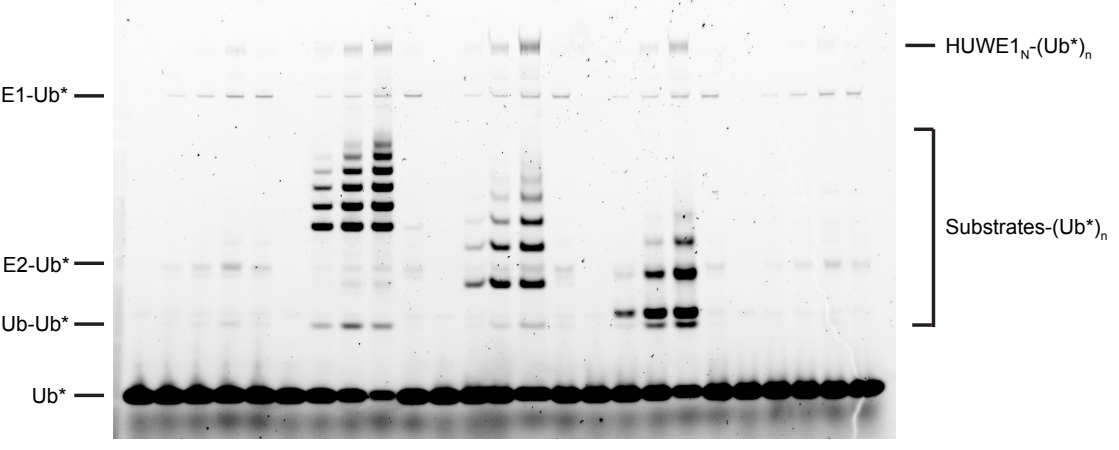

e Targeting of $\mathrm{H} 2 \mathrm{~B}$ and nucleosomes

Rpl26

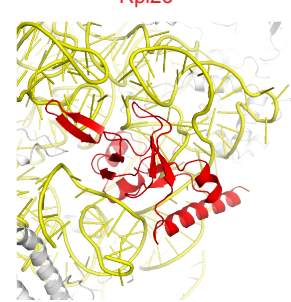

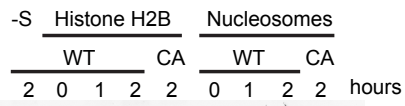

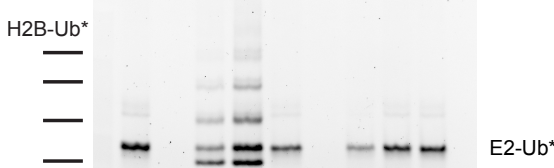

H2B

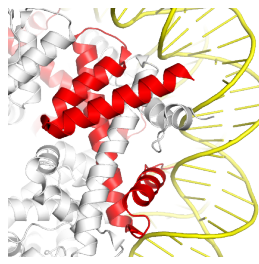

f DNA binding blocks Histone $\mathrm{H} 1$ ubiquitination

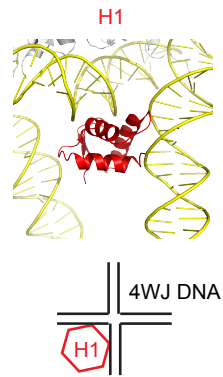

$2 \mu \mathrm{M}$ Histone $\mathrm{H} 1$

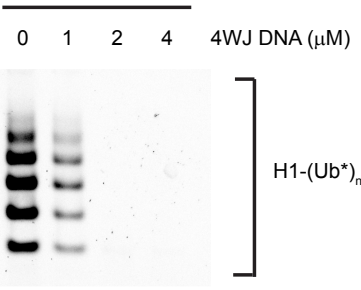

g Rpl26 ubiquitination sites

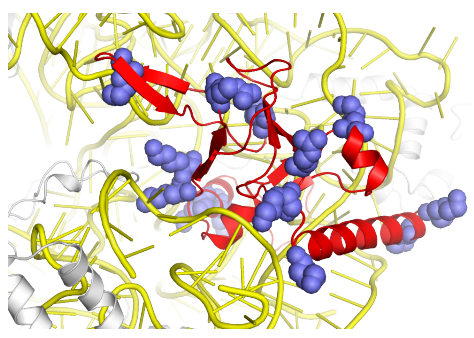

h C. elegans HUWE1 activity

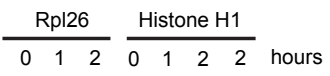

$$
\begin{aligned}
& \text { - } \quad \text { - } \quad \text { - } \quad \text { - + 4WJ DNA }
\end{aligned}
$$

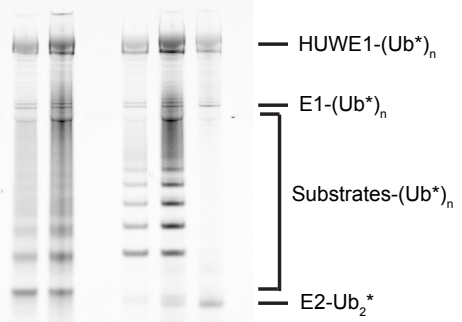


Figure 2

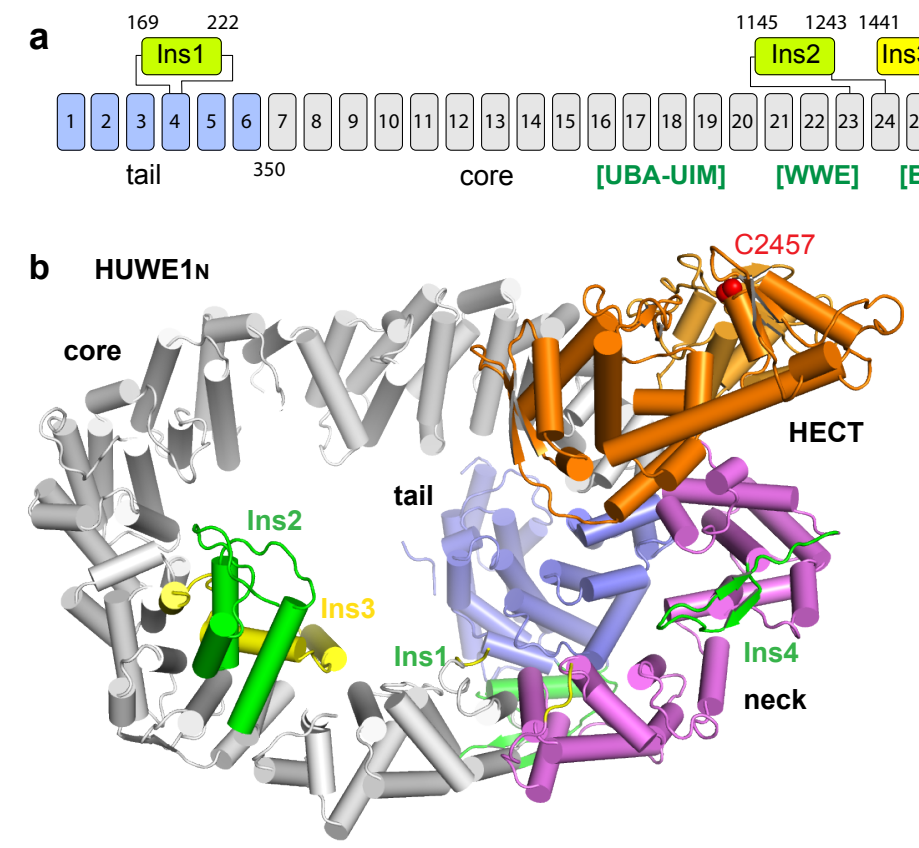

12 [ail 3

b HUWE1N

d CeHUWE1

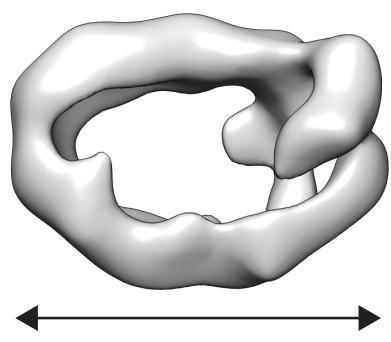

C

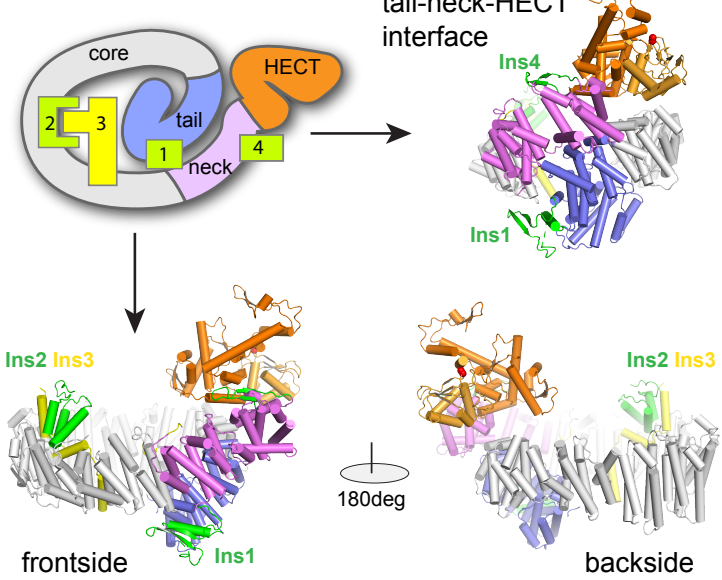

\section{d HUWE1 organization}

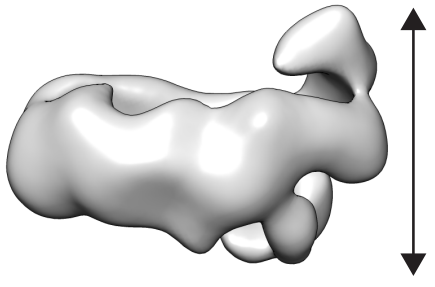

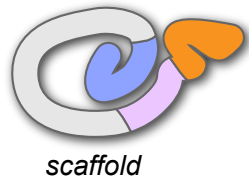

tail-core-neck HECT

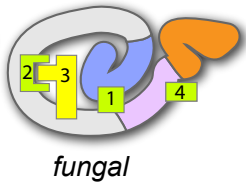

$+\operatorname{lns} 1+\operatorname{lns} 2$ +Ins3 +Ins4 (ring topology, S-targeting)

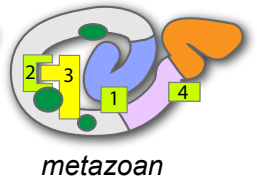

+ UBA + UIM $+\mathrm{WWE}+\mathrm{BH} 3$ (S-targeting) 


\section{Figure 3}

a Insertion Ins1

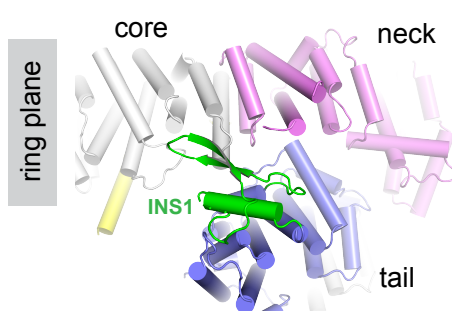

\section{b Insertion Ins2/3}

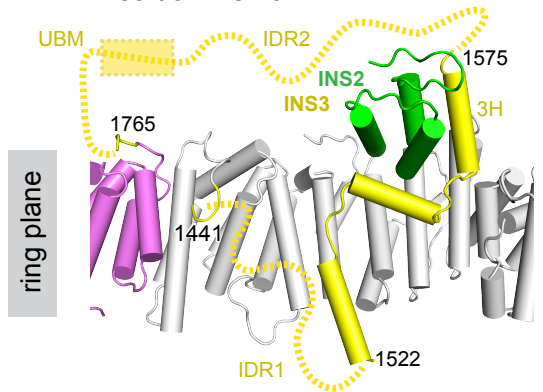

C Insertion Ins4

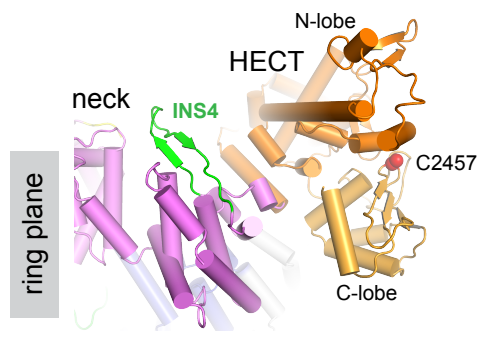

d Histone $\mathrm{H} 1$ ubiquitination

$\frac{\text { wild-type }}{0153060} \frac{\mathrm{CA}}{0153060} \frac{\Delta \mathrm{ins} 2}{0153060} \frac{\Delta 1500-1660}{0153060} \frac{\Delta \mathrm{UBM}}{0153060} \frac{\Delta \text { ins4 }}{0153060 \text { minutes }}$
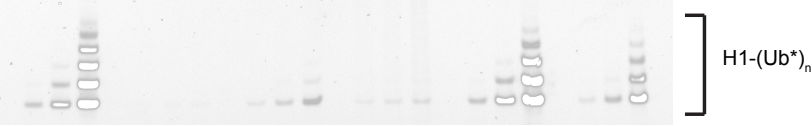

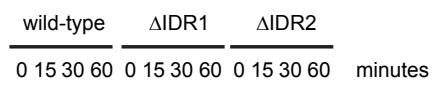

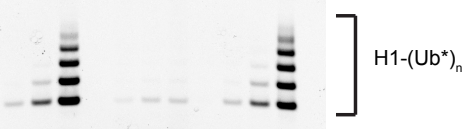

e Substrate-independent autoubiquitination

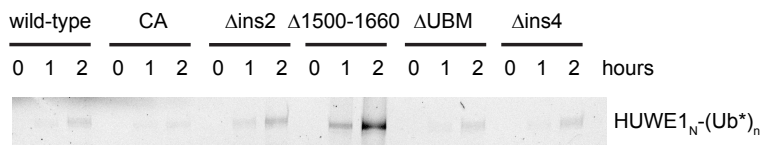

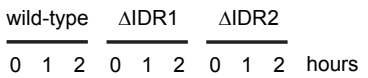

$$
\begin{aligned}
& \Leftrightarrow \quad \operatorname{HUWE} 1_{N}-\left(U b^{*}\right)_{n}
\end{aligned}
$$




\section{Figure 4}

a

HUWE1 ring interface
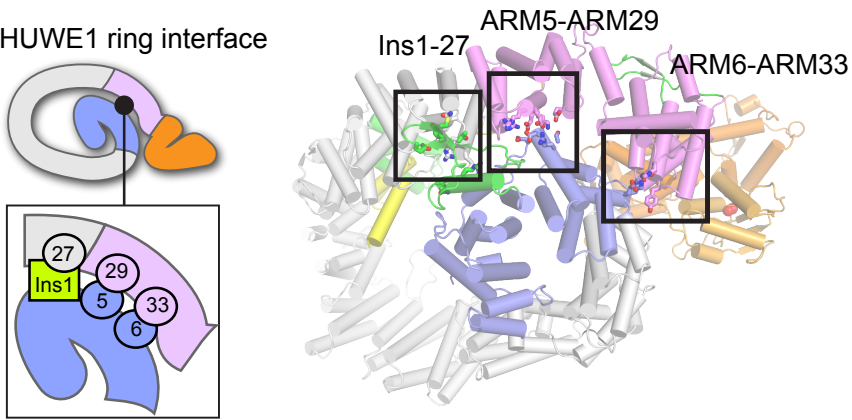

b

b Ins1-27
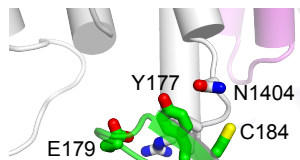

R1406
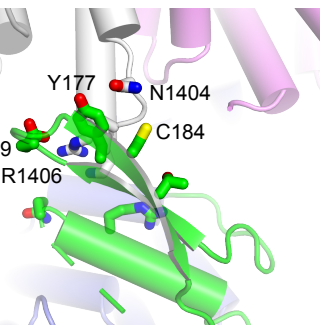

ARM6-ARM33*

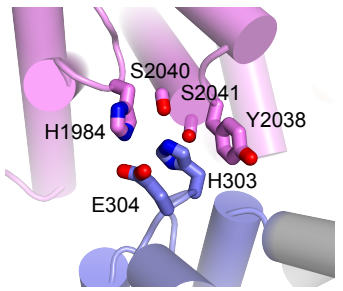

C Ring interface

$$
\text { Area }\left(\AA^{2}\right) \text { - H bonds - B-factor }\left(\AA^{2}\right)
$$

$\begin{array}{llll}\text { Ins1-27 } & 192 & 1 & 143 \\ 5-29 & 313 & 6 & 113 \\ 6-33 & 130 & 2 & 105\end{array}$


Figure 5

a XLMS analysis HUWE1N

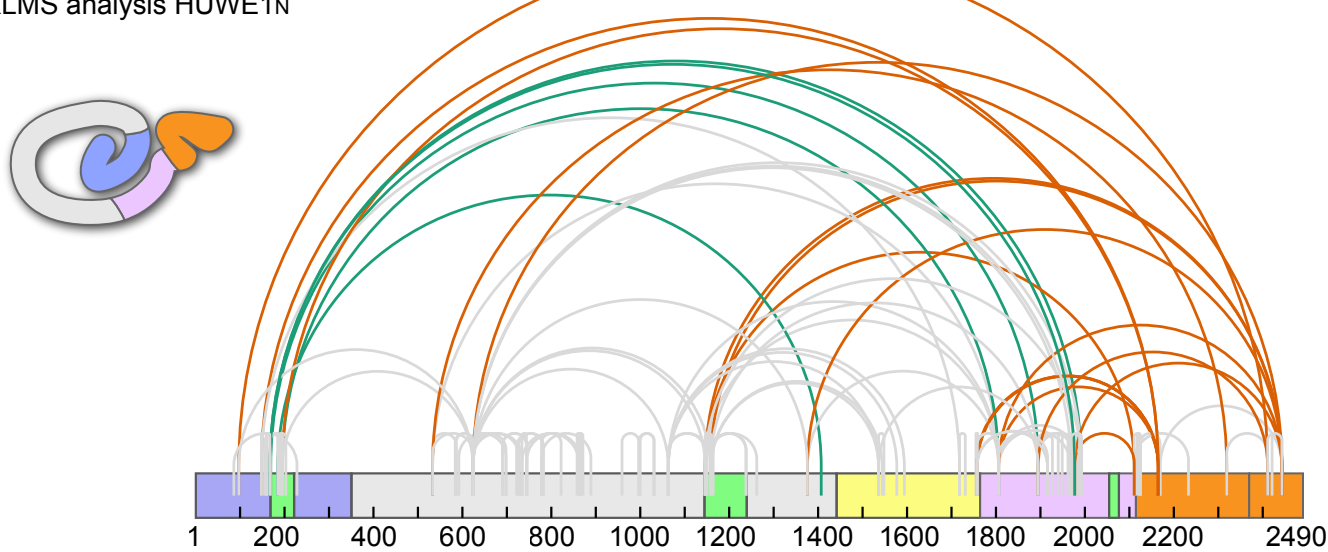

b CryoEM analysis

\& 20.

: 53 -

$\therefore$ L

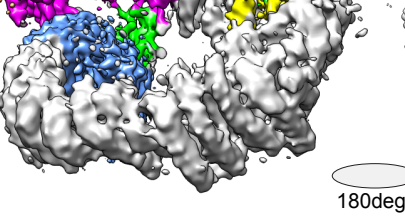

class 1

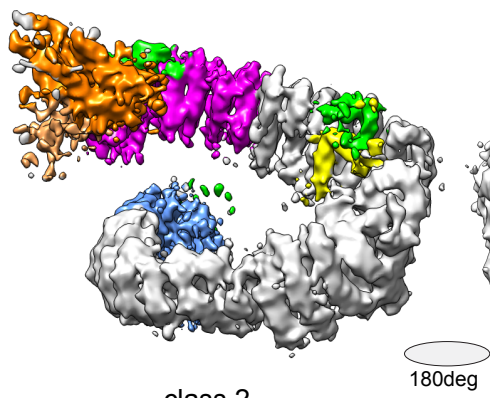

class 2

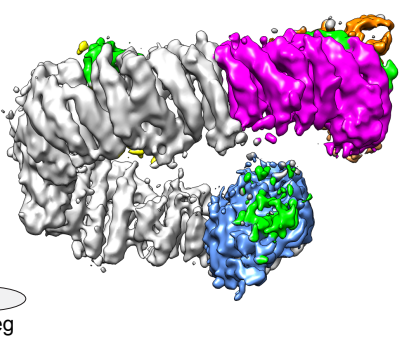

d

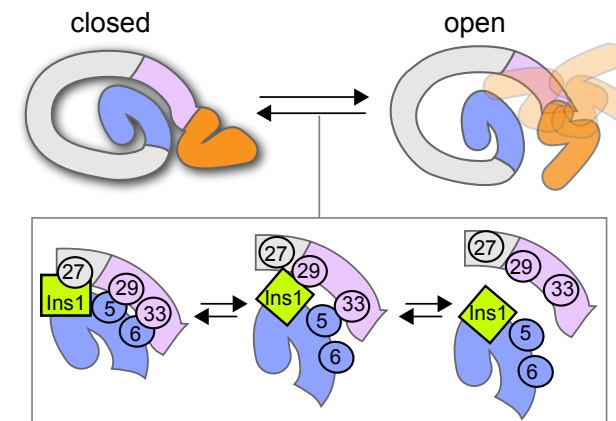

ring interface, between tail and neck

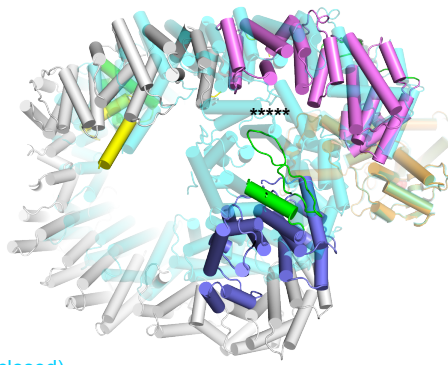

- HECT domain contacts ring interface contacts other crosslinks
Crystal structure (closed)

Partially open (class 1 )
Open solenoid (class 2)

c Opening of HUWE $1 \mathrm{~N}$

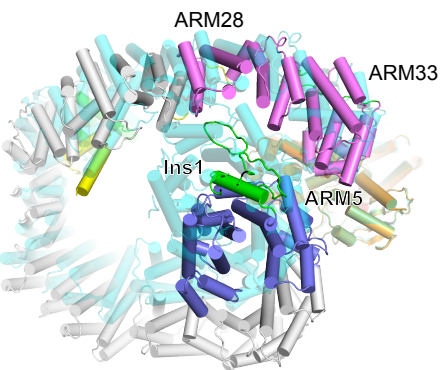


a separation of functional sites

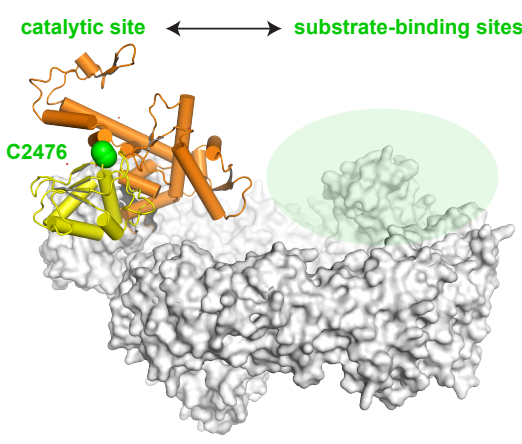

d Substrate recognition

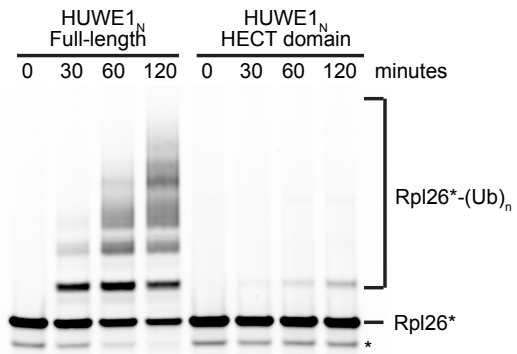
b binding of E2-Ub

UbcH5b-Ub / NEDD4L(HECT)

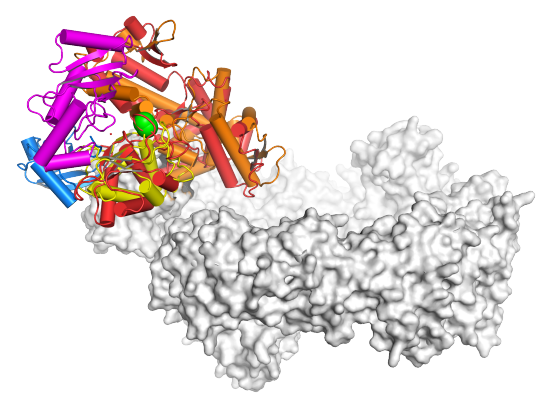

C Ub-charged HECT E3

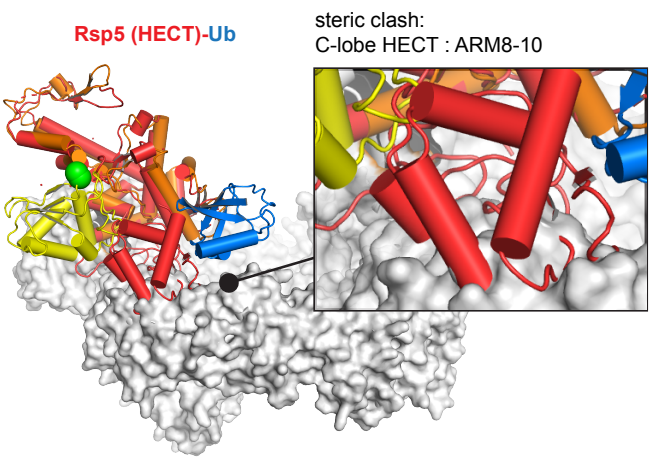

e Dysregulated autoubiquitination control

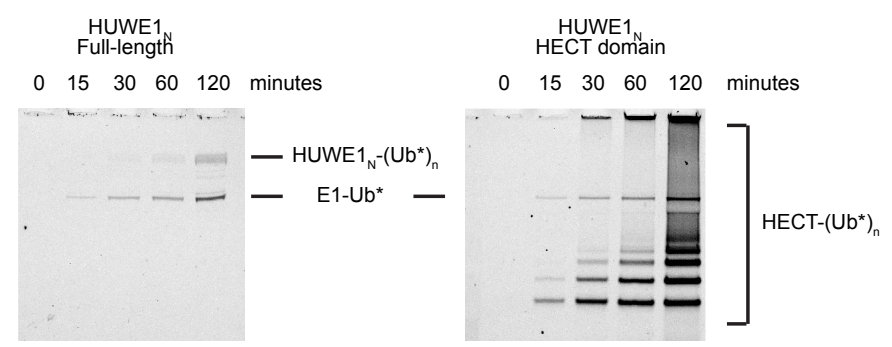

f Single turnover chase of loaded E2 by excess HUWE $1_{\mathrm{N}}$

Non-reducing - thioester and isopeptide $\mathrm{Ub}$

Reducing - only isopeptide Ub

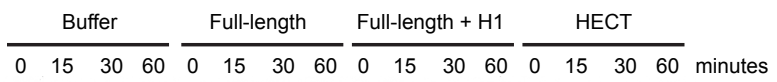

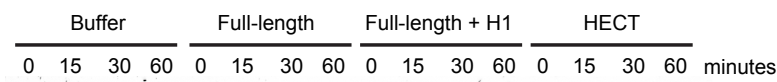

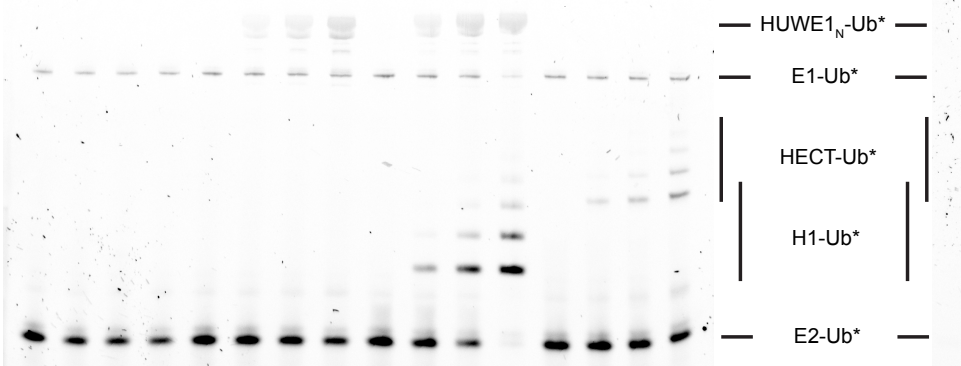

